\title{
Fate of drugs during wastewater treatment
}

Bruce Petrie $^{1}$, Ewan J. McAdam ${ }^{1}$, Mark D. Scrimshaw ${ }^{2}$, John N. Lester ${ }^{1}$ and Elise Cartmell $^{1}{ }^{*}$ ${ }^{1}$ Cranfield Water Science Institute, Cranfield University, Bedfordshire, MK43 0AL, UK. ${ }^{2}$ Institute for the Environment, Brunel University, Middlesex UB8 3PH, UK.

*corresponding author telephone: +44 (0)1234 758366 email: e.cartmell@cranfield.ac.uk

\begin{abstract}
Recent trends for the determination of pharmaceutical drugs in wastewaters focus on the development of rapid multi-residue methods. These encompass a large number of drugs (up to 90) of varying physicochemical composition (hydrophobicity, molecular weight etc) at ng $1^{-1}$ concentrations in the aqueous phase of complex heterogeneous matrices. These are well suited for drug determination in secondary effluents which contain detectable concentrations of pharmaceuticals. Drugs are not routinely monitored for in particulate phases of wastewaters despite being essential for fate determination, particularly in secondary processes receiving relatively high concentrations of suspended solids. Secondary effluents tend to contain multiple drugs, often above their proposed legislative targets for consent. Thus, tertiary processing may be considered to enhance drug removal and provide additional environmental protection. However, current analytical methods do not enable the efficacy of tertiary processes to be fully ascertained due to the inherently lower drug concentrations achieved. Existing method optimisation is required to lower detection capabilities for tertiary process monitoring. This would aid the understanding of breakdown reaction completeness and the criticality between parent drug and degradation product concentrations. Numerous degradation products are formed by biological and chemical processes which can exhibit toxicity. The complimentary use of biological assays here would improve understanding of the synergistic toxicological effect of multiple drugs and their degradation products at low concentration. Additionally, tertiary processes receive secondary effluents comprising comparatively high concentrations of dissolved organics (e.g., colloids). However, knowledge of drug behaviour in the charged colloidal fraction of wastewater and its impact to treatment is limited. Monitoring and understanding here is needed to develop tertiary process diagnosis and optimisation.
\end{abstract}

Key words: Pharmaceutical drug; $17 \alpha$-ethinylestradiol; metabolite; analytical method; liquid chromatography; mass spectrometry; activated sludge; sand filter; ozone; activated carbon 


\section{Introduction}

It is well established that wastewater contains a diverse range of anthropogenic compounds [1] and that their quantitative analysis poses numerous problems analytically, both in terms of extraction efficiency [2] and interference from co-extractives [3]. Through medical use large numbers of drugs are now included in this group of determinants [4-7]. A clear trend in legislation has been a lowering of acceptable environmental concentrations (e.g., 17 $\alpha$ ethinylestradiol (EE2)) and analytical method development has become an iterative process to achieve ever lower detection limits [8-9] as further potential environmental health issues emerge [10]. A growing understanding of the possible environmental impact to aquatic ecosystems has led to the classification of diclofenac and the steroid estrogen EE2 as priority hazardous chemicals [11-12]. These drugs have proposed environmental quality standards (EQS) of $100 \mathrm{ng} \mathrm{l}^{-1}$ and $0.035 \mathrm{ng} \mathrm{l}^{-1}$ respectively, and require monitoring to ensure compliance to 'good' water status [11-12]. The effects of EE2, which results in intersex in male fish, are the most studied of the drugs in the environment [13]. Concentrations of EE2 below $1 \mathrm{ng} \mathrm{l}^{-1}$ are known to cause intersex and vitellogenin induction in male fish during laboratory studies. The environmental impact of other drugs and mixtures is less clear. However, it has been demonstrated that a mixture of acetaminophen, carbamazepine, gemfibrozil and venlaflaxine (in the low $\mu \mathrm{g}^{-1}$ range) had a significant impact to fish embryo development in the short term [14]. The chronic impact of drugs (i.e., ecological and evolutionary), either individually or as mixtures, remains unknown [15]. Without such information it is desirable to limit entry of these chemicals into the aquatic environment. A total of 12 drugs of varying therapeutic class (ibuprofen, diclofenac, naproxen, ketoprofen, carbamazepine, bezafibrate, propranolol, fluoxetine, EE2, ofloxacin, erythromycin and oxytetracycline) are highlighted in this review to represent a variety of physicochemical compositions (e.g., molecular size, hydrophobicity) (Table 1). This includes those most studied in the literature [7, 17-18] and those in national studies (i.e., the UK Chemical Investigations Programme (CIP)) [19]. The CIP found diclofenac, propranolol, fluoxetine, EE2, erythromycin and oxytetracycline above their indicative legislative targets for consent $\left(10 \mathrm{ng} \mathrm{l}^{-1}\right.$ for those not listed as priority hazardous substances) in over $50 \%$ of effluents studied [19]. Consequently, a variety of drugs have been observed in surface waters in the $n g \mathrm{l}^{-1}$ range (Table 2). This underpins the need to understand drug fate during wastewater treatment for process/strategy optimisation.

Determining drug fate during wastewater treatment relies on the application of robust analytical methodologies. These require the ability to simultaneously determine a number of drugs, of differing physicochemical properties, within environmental matrices comprised of comparatively high concentrations of complex bulk organics [23]. The high complexity of environmental matrices underlines the analytical challenge posed. Drugs and their 
Table 1. Physicochemical properties of pharmaceutical drugs present in crude wastewaters and secondary effluents [16]

\begin{tabular}{|c|c|c|c|c|c|c|c|}
\hline Drug & $\begin{array}{c}\mathrm{MW} \\
/ \mathrm{g} \mathrm{mol}^{-1}\end{array}$ & $\begin{array}{c}\text { Water solubility } \\
\qquad / \mathrm{mg} \mathrm{l}^{-1}\end{array}$ & $\begin{array}{l}\text { Henry's law } \\
\text { / atm } \mathbf{m}^{3} \text { mol }^{-1}\end{array}$ & $\begin{array}{c}\mathrm{VP} \\
/ \mathrm{mm} \mathrm{Hg}\end{array}$ & pKa & $\log K_{o w}$ & $\log K_{o c}$ \\
\hline Ibuprofen & 206.30 & 21.0 & $1.52 .10^{-7}$ & $1.86 .10^{-4}$ & 4.91 & $3.79-3.97$ & 2.35 \\
\hline Diclofenac & 296.15 & 2.4 & $4.73 .10^{-12}$ & $2.18 .10^{-6}$ & 4.15 & $4.02-4.51$ & 2.61 \\
\hline Naproxen & 230.26 & 15.9 & $3.39 .10^{-10}$ & $1.27 .10^{-6}$ & 4.15 & $3.10-3.18$ & 1.97 \\
\hline Ketoprofen & 254.28 & 51.0 & $2.12 .10^{-11}$ & $6.81 .10^{-6}$ & 4.45 & $3.00-3.12$ & 2.08 \\
\hline Carbamazepine & 236.27 & 112 & $1.08 .10^{-10}$ & $8.80 .10^{-8}$ & - & $2.25-2.45$ & 2.23 \\
\hline Bezafibrate & 361.82 & 7.9 & $2.12 .10^{-15}$ & $6.12 .10^{-11}$ & - & 4.25 & 2.31 \\
\hline Propranolol & 259.35 & 61.7 & $7.98 .10^{-13}$ & $9.44 .10^{-8}$ & 9.42 & $2.60-3.48$ & 2.45 \\
\hline Fluoxetine & 309.33 & 60.3 & $8.90 .10^{-8}$ & $2.52 .10^{-5}$ & - & $4.05-4.65$ & 4.97 \\
\hline EE2 & 296.40 & 11.3 & $7.94 .10^{-12}$ & $1.95 .10^{-9}$ & - & $3.67-4.12$ & 4.65 \\
\hline Ofloxacin & 361.37 & $2.8 .10^{4}$ & $4.98 .10^{-20}$ & $9.84 .10^{-13}$ & - & $<0$ & 1.09 \\
\hline Erythromycin & 733.94 & 0.5 & $5.42 .10^{-29}$ & $2.12 .10^{-25}$ & 8.88 & $2.48-3.06$ & 2.75 \\
\hline Oxytetracycline & 460.43 & 313 & $1.70 .10^{-25}$ & $9.05 .10^{-23}$ & 3.27 & $<0$ & 1.87 \\
\hline
\end{tabular}

KEY: MW, molecular weight; VP, vapour pressure; $\mathrm{pKa}$, acid dissociation constant; Log $\mathrm{K}_{\mathrm{ow}}$, octanolwater coefficient; $\log \mathrm{K}_{\mathrm{oc}}$, organic carbon-water coefficient

metabolites were first reported in wastewater effluents in the 1970's [24]. Hignite et al [24] measured chlorophenoxyisobutyrate and salicylic acid in wastewater effluents at relatively high mean concentrations of $7 \mu \mathrm{g}^{-1}$ and $29 \mu \mathrm{g} 1^{-1}$, respectively. Ion exchange chromatography was used for extraction followed by gas chromatography-mass spectrometry (GC-MS). Numerous other drugs have been given significant attention recently due to the development of sophisticated analytical methodologies [17-18, 25]. Extraction media enabling simultaneous retention of acidic, basic and neutral species of varying polarities has aided this. Furthermore, the coupling of liquid chromatography (LC), and particularly ultraperformance liquid chromatography (UPLC) to MS has seen significant reductions in sample pre-treatment requirements and instrument analysis time [23]. Other than analytical difficulties, the variety of physicochemical behaviour (e.g., hydrophobicity) exhibited by drugs results in significant differences in their fate and removal during wastewater treatment $[6,26]$. Tertiary treatment processes are being considered to enhance hazardous chemical removal to levels which comply with proposed EQS's [27], as are the analytical methods capable of supporting their diagnosis and optimisation for this critical group of emerging chemicals. This review addresses recent analytical trends for drug determination in environmental matrices used to facilitate fate studies. Analytical requirements for further fate evaluation and tertiary process selection/optimisation are also discussed. 
Table 2. Recently reported occurrence of pharmaceutical drugs in surface waters

\begin{tabular}{|c|c|c|c|c|c|}
\hline $\begin{array}{l}\text { Drug of } \\
\text { interest }\end{array}$ & Class & Chemical structure & $\begin{array}{c}\text { Proposed } \\
\text { legislative } \\
\text { target / } \text { ng l }^{-1} \\
\end{array}$ & $\begin{array}{c}\text { Surface } \\
\text { water }^{\mathrm{a}} / \mathrm{ng} \mathrm{l}^{-1}\end{array}$ & Location \\
\hline Ibuprofen & NSAID & & $10^{\mathrm{b}}$ & $\begin{array}{c}<0.3-56 \\
<6.4-542 \\
21-2,796 \\
\end{array}$ & $\begin{array}{c}\text { UK } \\
\text { Mainland Europe } \\
\text { North America }\end{array}$ \\
\hline Diclofenac & NSAID & & $100^{\mathrm{c}}$ & $\begin{array}{c}<0.5-261 \\
<12-154 \\
17-42\end{array}$ & $\begin{array}{c}\text { UK } \\
\text { Mainland Europe } \\
\text { North America }\end{array}$ \\
\hline Naproxen & NSAID & & - & $\begin{array}{c}<0.3-55 \\
<3.1-109 \\
22 \\
\end{array}$ & $\begin{array}{c}\text { UK } \\
\text { Mainland Europe } \\
\text { North America }\end{array}$ \\
\hline Ketoprofen & NSAID & & - & $\begin{array}{c}<0.5-4 \\
<15-517 \\
-\end{array}$ & $\begin{array}{c}\text { UK } \\
\text { Mainland Europe } \\
\text { North America }\end{array}$ \\
\hline Carbamazepine & Anti-epileptic & & - & $\begin{array}{l}0.5-495 \\
<1.5-54 \\
1-1,238\end{array}$ & $\begin{array}{c}\text { UK } \\
\text { Mainland Europe } \\
\text { North America }\end{array}$ \\
\hline Bezafibrate & $\begin{array}{l}\text { Lipid } \\
\text { regulator }\end{array}$ & & - & $\begin{array}{c}<10-66 \\
<2.0-26 \\
-\end{array}$ & $\begin{array}{c}\text { UK } \\
\text { Mainland Europe } \\
\text { North America }\end{array}$ \\
\hline Propranolol & Beta blocker & & $10^{\mathrm{b}}$ & $\begin{array}{c}<0.5-27 \\
<0.4-39 \\
53\end{array}$ & $\begin{array}{c}\text { UK } \\
\text { Mainland Europe } \\
\text { North America }\end{array}$ \\
\hline Fluoxetine & $\begin{array}{c}\text { Anti- } \\
\text { depressant }\end{array}$ & & $10^{\mathrm{b}}$ & $\begin{array}{c}- \\
<7.4-24 \\
<1.3-65\end{array}$ & $\begin{array}{c}\text { UK } \\
\text { Mainland Europe } \\
\text { North America }\end{array}$ \\
\hline EE2 & Contraceptive & & $0.035^{\mathrm{c}}$ & $\begin{array}{l}- \\
- \\
-\end{array}$ & $\begin{array}{c}\text { UK } \\
\text { Mainland Europe } \\
\text { North America }\end{array}$ \\
\hline Ofloxacin & Antibiotic & & $10^{\mathrm{b}}$ & $\begin{array}{c}- \\
4.8-105 \\
-\end{array}$ & $\begin{array}{c}\text { UK } \\
\text { Mainland Europe } \\
\text { North America }\end{array}$ \\
\hline Erythromycin & Antibiotic & & $10^{\mathrm{b}}$ & $\begin{array}{l}<0.5-20 \\
<28-52 \\
2-438\end{array}$ & $\begin{array}{c}\text { UK } \\
\text { Mainland Europe } \\
\text { North America }\end{array}$ \\
\hline Oxytetracycline & Antibiotic & & $10^{\mathrm{b}}$ & $\begin{array}{c}- \\
<12-37 \\
-\end{array}$ & $\begin{array}{c}\text { UK } \\
\text { Mainland Europe } \\
\text { North America }\end{array}$ \\
\hline
\end{tabular}

a occurence data taken from: UK-[20] Mainland Europe-[17] North America-[21-22], ${ }^{b}$ UK Chemicals Investigation Programme [19], ${ }^{\mathrm{c}}[12]$

KEY: NSAID, non steroidal anti-inflammatory drug 


\section{Analytical strategies for fate evaluation}

Prior to laboratory work, the first step for drug determination in wastewaters is sampling. This process is fundamental to any strategy for monitoring, and careful consideration of sampling equipment, sample handling and types of samples is needed. It is beyond the scope of this review to examine further; however an excellent overview of sampling strategies has been given by Ort et al [28-29]. Nevertheless, it is important to highlight that to fully understand fate during wastewater treatment, determination in both aqueous and particulate phases of wastewaters is essential $[9,30]$.

Trace determination of drugs in aqueous wastewater fractions (typically $0.45 \mu \mathrm{m}$ filtered) requires an enrichment step followed by chromatographic separation and mass spectrometry (MS) detection. Sample pre-concentration and clean up commonly involves solid phase extraction (SPE), and can be undertaken off-line (using extraction systems not linked to analytical equipment) or on-line, where extraction and quantification are automated and linked together. Off-line analysis tends to use up to 1 litre of sample, and Gros et al [31] investigated the efficacy of various SPE sorbents (Oasis HLB, Oasis MCX, Isolute C18 and Isolute $\mathrm{ENV}+$ ) for the simultaneous extraction of 13 pharmaceutical drugs of varying physicochemical composition. The Oasis HLB sorbent (polystyrene-devinylbenzene-Nvinylpyrrolidone terpolymer) [32] (without $\mathrm{pH}$ adjustment) exhibited superior performance, utilising both hydrophilic and lipophilic retention mechanisms. Consequently, a full suite of drugs (up to 90) can be extracted simultaneously off-line using this sorbent [17-18, 25, 33]. The introduction of fully automated methods further reduces sample processing restrictions [34-35]. At present these are emerging techniques whose use are not widespread. Their reproducibility and ability to use smaller total sample volumes mean they will supersede traditional labour intensive SPE protocols in commercial laboratories in the future. Drug determination in the particulate phase of wastewaters is not routinely monitored but those methods which do, use ultrasonic solvent extraction (USE) [36] or most commonly, accelerated solvent extraction (ASE) [30, 37-40]. The application of pressure enables the use of extraction solvents (eg methanol) at temperatures much higher than their boiling point, increasing solubility and mass transfer [41]. Following extraction the solvent can be diluted in water to $<5 \%(\mathrm{v} / \mathrm{v})$ and subjected to SPE as an aqueous fraction [38-39].

Gas chromatography-mass spectrometry is well established for quantification of chemicals in environmental samples, achieving method quantitation limits (MQLs) $\leq 10 \mathrm{ng} \mathrm{l}^{-1}$ for some drugs [42-43]. However, derivatization of more polar drugs with toxic chemicals is required prior to analysis to improve volatility, thermal stability and sensitivity of detection, increasing cost and time of sample preparation. A further disadvantage is the run time required for 
analysis; often up to 1 hour per sample [42-43]. This has been a rate limiting step of such research in the past, severely restricting sample numbers which can be analysed. Despite these limitations, methods report the ability to simultaneously measure $\geq 63$ drugs of varying therapeutic class from a single injection by GC-MS following derivatization [44-45]. The use of LC coupled to tandem MS detection (MS/MS) improves sample throughput without the need for additional sample preparation [46]. Furthermore, the introduction of UPLC offers additional reductions in run times, whilst improving sensitivity over conventional LC [23]. For UPLC, run times are generally less than 10 minutes with MQLs $<100 \mathrm{ng} \mathrm{l}^{-1}$ for most drugs [17-18, 25]. However, a well known problem of environmental sample analysis by LCMS, with electrospray ionisation (ESI) source particularly, is the quenching influence (ionisation suppression) of sample matrix on analyte signal strength [23, 47]. The commercial availability of deuterated surrogates now offers substantial improvements in minimizing the impact of matrix interferences, improving accuracy of analysis and up to 50 isotopic labelled standards are used in some multi-residue methods [35].

The selection of MS/MS detector type is critical for analysis type (i.e., qualitative or quantitative). Quantitative analysis commonly employs a triple quadrupole (QqQ) due to its high sensitivity. The use of hybrid detectors such as quadrupole time of flight (QqTOF) offers the ability to screen and identify unknown degradation products/metabolites. Its full scan sensitivity, high selectivity and specificity enable structural elucidation of non-target species [32]. This is a significant advance in the determination of drug fate where degradation products in both biological and chemical processes are formed; enabling degradation pathways to be identified. However, data processing can be time consuming due to the lack of searchable libraries, often requiring manual spectral interpretation [32, 46]. A further disadvantage of TOF detection is it generally offers lower sensitivity ( 3 to 5 times) than conventional MS/MS such as QqQ [48]. Thus, the ever increasing requirement to reduce MQLs for trace analysis confines its use to qualitative fate evaluation. Alternatively, hybrid linear ion trap (LIT) Orbitrap instrumentation offers high sensitivity for environmental quantitation (as offered by QqQ) and the ability to perform accurate mass determinations for drug identification (as offered by TOF) [46]. Although Orbitrap technology has been used to screen environmental samples for unknowns [49], unequivocal confirmation of degradation products observed by non-target MS/MS screening requires a complementary analytical technique or use of analytical reference standards [50]. However, as degradation products are often not known, there is a subsequent lack of standards for these. Further opportunities are offered by Fourier transform-MS which demonstrates unrivalled mass accuracy, rapid data collection, good dynamic range and, high sensitivity and resolution. However, the high cost of such instrumentation limits its widespread application. 
Overall, recent trends for drug determination in wastewaters tend to use a single stage Oasis HLB off-line SPE followed by UPLC with detection by QqQ [17, 25, 33] or LIT [18] (Table $3)$. These methods are well suited for the determination of multiple drugs in the aqueous phase of crude wastewaters and secondary effluents where their concentrations are relatively high. However, monitoring EE2 at environmentally relevant levels requires devoted clean up protocols which can be laborious due to its inherently lower concentrations [23]. Nevertheless, there is a lack of particulate phase analysis undertaken and the concentrations present in tertiary effluents continue to pose an analytical challenge.

\section{Drug removal by conventional wastewater treatment; the current problem}

Activated sludge is an extensively implemented secondary wastewater treatment process, effective for carbonaceous material removal and can be adapted for nutrient removal. Removals of many drugs (and other chemicals of anthropogenic origin) are also observed [67]. Removal of drugs and other hazardous chemicals during treatment is attributed to biological degradation and sorption onto biomass [52-54]. Pharmaceutical drugs have low vapour pressures and pKa's ranging from 3 to 10 (Table 1) restricting any removal by volatilization. The relative resistance to biodegradation and/or sorption of some drugs makes enhancing removal by such processes difficult. Ibuprofen, diclofenac and carbamazepine encompass extremities in susceptibility to biodegradation and sorption. These represent drugs reasonably amenable to sorption and biodegradation (ibuprofen), sorption only (diclofenac) and neither sorption or biodegradation (carbamazepine), respectively. Consequently, removal differs between one another during activated sludge treatment [6-7]. Solids retention time (SRT) (which is proportional to the food: micro-organism (F:M) ratio) is the simplest way of manipulating existing activated sludge operation in the short term and, is considered critical to the removal of non-drug derived hazardous chemicals [6-7, 27]. An increased SRT (>10 days) is often cited as the condition required to achieve greatest hazardous chemical removal [6-7] but has little effect to removals of these drugs (Figure 1, Table 4). Generally, diclofenac is removed by $\leq 50 \%$ and any carbamazepine removal is negligible. Negative drug removals are also observed during treatment and are considered attributable to the deconjugation of metabolites present in the crude stream [26]. Conjugates and intermediate chemicals tend to go undetermined by current analytical approaches. Parent drugs are often above their legislative targets for consent in secondary effluents despite accounting for typical dilution ratios in the environment [19]. Source control would limit drug entry into wastewater, similar to what has been achieved with nonylphenol [23]. Without the availability of substitute drugs, less persistent and with a less burdensome environmental impact, this will not be achievable. However, the possibility of separate treatment of urine 
Table 3. Recently validated LC-MS/MS methods applied for the quantitation of drugs in environmental matrices

\begin{tabular}{|c|c|c|c|c|c|c|c|c|c|c|c|c|c|c|}
\hline \multirow{2}{*}{$\begin{array}{l}\text { No. of } \\
\text { drugs }\end{array}$} & \multirow{2}{*}{ Sample } & \multirow{2}{*}{ SPE } & \multirow{2}{*}{ Chromatography } & \multirow{2}{*}{ Detector } & \multirow{2}{*}{$\begin{array}{l}\text { Run } \\
\text { time / } \\
\text { min }^{-1}\end{array}$} & \multirow{2}{*}{$\begin{array}{l}\text { SE } \\
\text { recovery } \\
/ \%\end{array}$} & \multirow{2}{*}{$\begin{array}{l}\text { SE MQL } \\
/ \mathrm{ng} \mathrm{l}^{-1}\end{array}$} & \multicolumn{4}{|c|}{$\begin{array}{c}\text { No. of drugs quantified in } \\
\text { methods application }\end{array}$} & \multirow{2}{*}{$\begin{array}{l}\text { Method } \\
\text { benefits }\end{array}$} & \multirow{2}{*}{$\begin{array}{l}\text { Method } \\
\text { limitations }\end{array}$} & \multirow{2}{*}{ Ref. } \\
\hline & & & & & & & & $\begin{array}{l}\text { Crude } \\
\text { WW }\end{array}$ & SE & $\mathbf{T E}$ & SW & & & \\
\hline 81 & Aq. & Oasis HLB & UPLC & QqLIT & $\begin{array}{l}4^{\mathrm{a}} \\
7^{\mathrm{b}}\end{array}$ & $22-146$ & $0.6-51$ & $57^{\mathrm{c}}$ & 59 & 28 & 45 & $\sqrt{ }$ & $\mathrm{XX}$ & [18] \\
\hline 47 & Aq. & Oasis HLB & UPLC & QqQ & 10 & $49-127$ & $0.8-170$ & - & 37 & - & 31 & $\sqrt{ }$ & $\mathrm{X}$ & [25] \\
\hline 74 & Aq. & Oasis HLB & UPLC & QqQ & $\begin{array}{l}8^{\mathrm{a}} \\
5^{\mathrm{b}}\end{array}$ & $0-174$ & $0.1-378$ & - & - & - & 73 & $\sqrt{ }$ & $\begin{array}{c}\mathrm{X} \\
\mathrm{XX}\end{array}$ & [17] \\
\hline 90 & Aq. & Oasis HLB & HPLC & QqQ & 25 & $5-246$ & $0.1-78$ & - & 63 & - & - & $\sqrt{ }$ & $\mathrm{X}$ & [33] \\
\hline $33^{\mathrm{d}}$ & Aq. & $\begin{array}{l}\text { Oasis HLB } \\
+3 \text { sorbents }\end{array}$ & HPLC & QqQ & $36^{\mathrm{e}}$ & $64-166$ & $2.3-186$ & - & - & - & 16 & $\begin{array}{l}\sqrt{ } \\
\sqrt{ } \sqrt{ }\end{array}$ & $X$ & {$[35]$} \\
\hline 87 & Aq. & $\begin{array}{l}\text { TurboFlow } \\
\text { column }\end{array}$ & HPLC & QqQ & $22^{\mathrm{e}}$ & $12-345$ & $0.1-164$ & - & - & - & 44 & $\begin{array}{l}\sqrt{ } \\
\sqrt{ } \\
\sqrt{ } \sqrt{ }\end{array}$ & $\begin{array}{c}\mathrm{X} \\
\mathrm{XX}\end{array}$ & [34] \\
\hline 14 & Part. & Oasis HLB & HPLC & QqQ & - & $70-120$ & $0.6-19^{\mathrm{f}, \mathrm{g}}$ & - & $11^{\mathrm{g}}$ & - & - & $\sqrt{ } \sqrt{ } \sqrt{ }$ & $\mathrm{X}$ & [40] \\
\hline 60 & Part. & Oasis MCX & UPLC & QqQ & 20 & $7-142^{\mathrm{e}}$ & $0.1-20^{\mathrm{f}, \mathrm{g}}$ & $30^{\mathrm{f}}$ & - & - & - & $\sqrt{ }$ & $\mathrm{X}$ & [30] \\
\hline 5 & $\begin{array}{l}\text { Aq. } \\
\text { Part. }\end{array}$ & MIP & HPLC & QqQ & 25 & $\begin{array}{c}95-105 \\
77-91\end{array}$ & $\begin{array}{c}4-12 \\
4-10^{\mathrm{f}, \mathrm{i}}\end{array}$ & - & $5^{\mathrm{j}}$ & - & $4^{\mathrm{f}, \mathrm{i}}$ & $\sqrt{ } \sqrt{ } \sqrt{ }$ & $\begin{array}{c}\mathrm{X} \\
\mathrm{XXX}\end{array}$ & [51] \\
\hline $\begin{array}{c}1^{\mathrm{k}} \\
(\mathrm{EE} 2)\end{array}$ & $\begin{array}{l}\text { Aq. } \\
\text { Part. }\end{array}$ & $\begin{array}{c}\mathrm{C} 18, \mathrm{NH}^{2} \\
\text { Silica, } \mathrm{NH}^{2}\end{array}$ & UPLC & QqQ & 9 & $\begin{array}{l}96 \\
97 \\
\end{array}$ & $\begin{array}{l}0.06 \\
2.96^{\mathrm{f}}\end{array}$ & 1 & 1 & - & - & $\begin{array}{l}\sqrt{ } \sqrt{ } \sqrt{ } \\
\sqrt{ } \sqrt{ } \sqrt{ } \sqrt{ }\end{array}$ & $\begin{array}{c}\mathrm{X} \\
\mathrm{XXXX}\end{array}$ & [23] \\
\hline \multicolumn{15}{|c|}{ 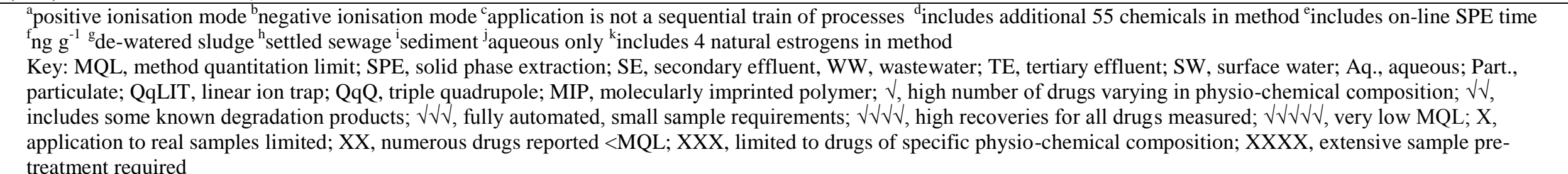 } \\
\hline
\end{tabular}




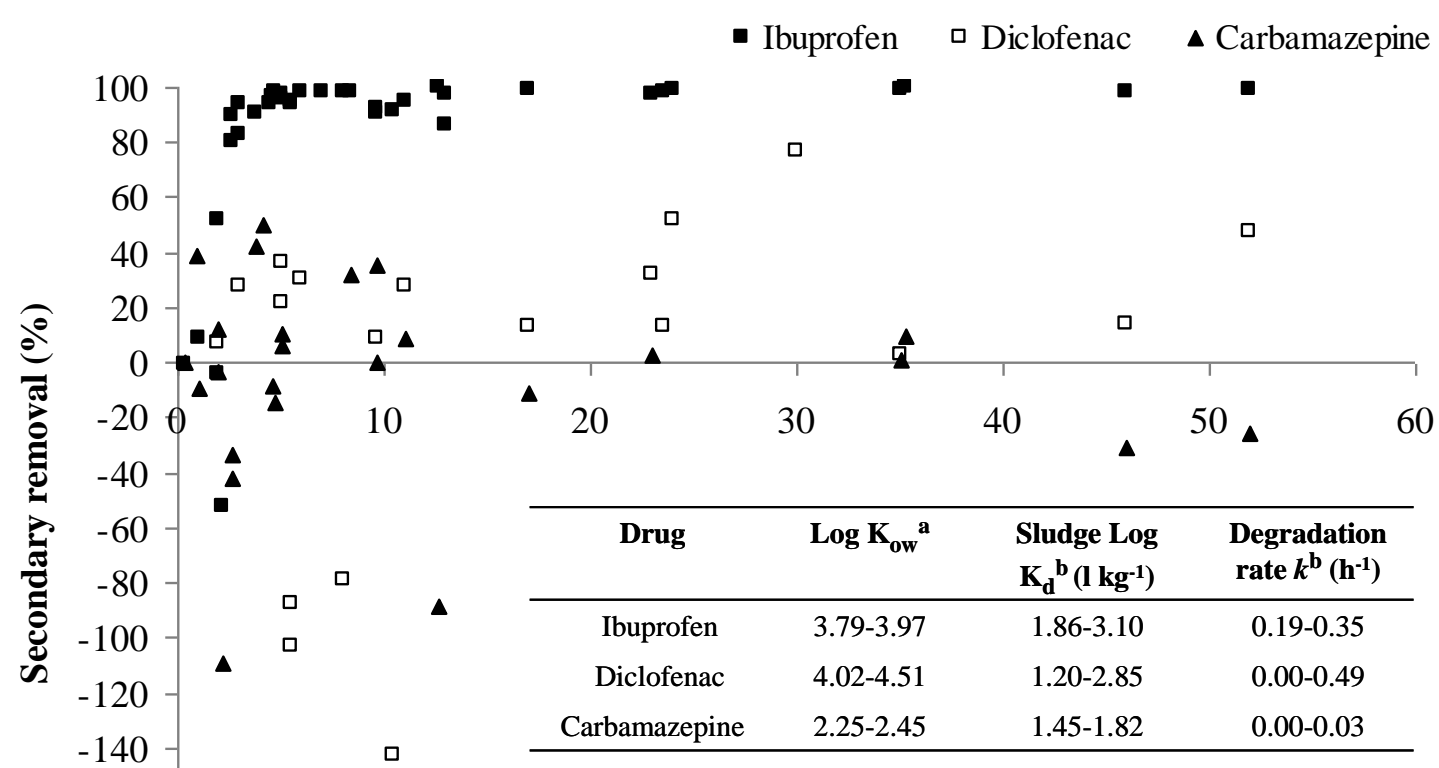

Solids retention time (SRT, days)

Figure 1. Removals of ibuprofen $(\square, n=36)$, diclofenac $(\square, n=19)$ and carbamazepine $(\Delta, n=25)$ by activated sludge operating at varying solids retention time. Ibuprofen, diclofenac and carbamazepine represent drugs reasonably amenable to sorption and biodegradation, sorption only and neither sorption or biodegradation, respectively. Inset, octanol-water coefficients, partition coefficients and degradation rate constants $-{ }^{\mathrm{a}}[16]{ }^{\mathrm{b}}[53]$ (removal data obtained from: [6-7, 26, 55-62] - Table 4, no data reported as $<$ MDL)

streams may be an effective solution in some circumstances [63]. Additionally, membrane bioreactor systems generally achieve greater drug removals than conventional secondary processes [32, 60-61]. Without their application, the remaining alternative to enhance drug removal is the addition of a tertiary process or processes to an existing conventional secondary treatment asset (e.g., activated sludge). This requires a process not excessively space consuming and can treat secondary effluents at relatively short contact times. Some available options suiting these criteria include; biofiltration (sand or trickling filters), chemical (ozone) and adsorption (granular activated carbon) processes. The fate and removal of drugs differ substantially between these systems.

\section{Drug fate and removal in tertiary processes}

\subsection{Biologically active sand filters}

Drug removal by biofiltration processes (tertiary sand or trickling filters) is by physical and biological mechanisms. Total removals by sand filters vary from $2 \%$ for carbamazepine to $>95 \%$ for ibuprofen [64] (Figure 2, Table 5), similar to those achieved by activated sludge. Despite treating different wastewaters of differing composition, activated sludge and tertiary sand filters essentially rely on the same mechanisms. Sand filters depend on a fixed biofilm 
Table 4. Removal of ibuprofen, diclofenac and carbamazepine in activated sludge at varying SRT

\begin{tabular}{|c|c|c|c|c|c|c|c|c|c|}
\hline Drug & $\begin{array}{c}\text { Flow rate / } \\
\mathbf{m}^{3} \mathbf{d}^{-1}\end{array}$ & SRT/ $d^{-1}$ & $\underset{1}{\text { HRT/h }}$ & $\begin{array}{c}\text { MLSS / } \\
\mathrm{mg} \mathrm{l}^{-1}\end{array}$ & $\begin{array}{l}\mathrm{COD} / \\
\mathrm{mg} \mathrm{l}^{-1}\end{array}$ & $\begin{array}{l}\text { SS conc. } \\
/ \mathrm{ng} \mathrm{l}^{-1}\end{array}$ & $\begin{array}{c}\text { SE conc. } \\
/ \mathrm{ng} \mathrm{l}^{-1}\end{array}$ & $\begin{array}{c}\text { Removal } \\
/ \%\end{array}$ & Ref. \\
\hline \multirow[t]{36}{*}{ Ibuprofen } & - & 0.3 & - & - & - & - & - & -1.0 & [6] \\
\hline & - & 1 & - & - & - & - & - & 9.0 & [6] \\
\hline & 626,000 & 1.9 & 7 & - & - & 50,700 & 24,600 & 51.5 & [55] \\
\hline & - & 2 & 1.9 & 4,000 & - & 2,300 & 2,400 & -4.2 & [7] \\
\hline & 47,860 & 2.2 & 13 & - & - & 14,200 & 21,700 & -52.8 & [55] \\
\hline & 185,000 & 2.7 & 12 & - & - & 27,900 & 5,400 & 80.6 & [55] \\
\hline & 585,667 & 2.7 & 14 & - & - & 58,200 & 6,200 & 89.3 & [55] \\
\hline & 3,967 & 3 & 10 & 3,030 & $113^{*}$ & - & - & 94.0 & [26] \\
\hline & 22,000 & 3 & 12 & - & 508.2 & $\begin{array}{c}13,355- \\
17,585\end{array}$ & $\begin{array}{l}1,420- \\
6,056\end{array}$ & 82.5 & [61] \\
\hline & 645,000 & 3.8 & 8.6 & - & - & 909 & 86.7 & 90.5 & [58] \\
\hline & 1,984 & 4.5 & 15 & 2,482 & $113^{*}$ & - & - & 94.0 & [26] \\
\hline & 409,000 & 4.6 & 8.0 & - & - & 593 & 21.0 & 96.4 & [58] \\
\hline & 125,248 & 4.7 & 13 & - & - & 21,800 & 300 & 98.6 & [55] \\
\hline & $1,199,000$ & 5.0 & 7.1 & - & - & 578 & 14.3 & 97.5 & [58] \\
\hline & - & 5.0 & - & - & - & - & - & 96.0 & [6] \\
\hline & 5,506 & 5.5 & 15 & 2,836 & $205^{*}$ & - & - & 95.0 & [26] \\
\hline & 15,300 & 5.5 & 15 & 1,743 & $154^{*}$ & - & - & 94.0 & [26] \\
\hline & 19,260 & 6 & 22 & 2,084 & $128^{*}$ & - & - & 98.0 & [26] \\
\hline & 125,000 & 7 & 12 & 2,000 & - & 1,966 & 40.0 & 98.0 & [60] \\
\hline & 20,000 & $6-10$ & 35 & - & $510-680$ & 5,700 & 88.5 & 98.4 & [62] \\
\hline & 210,000 & 8.4 & 8.9 & - & - & 595 & 8.0 & 98.7 & [58] \\
\hline & 68,498 & 9.6 & 15 & - & - & 27,300 & 2,700 & 90.1 & [55] \\
\hline & - & 9.6 & - & - & - & - & - & 92.0 & [6] \\
\hline & 17,994 & 10.5 & 13 & 2,105 & $51 *$ & - & - & 91.0 & [26] \\
\hline & - & $10-12$ & 7.3 & - & - & - & - & $91-99$ & [56] \\
\hline & 366,898 & 12.6 & 23 & - & - & 39,100 & 50.0 & $>99.9$ & [55] \\
\hline & 60,000 & $12-14$ & 22 & $\begin{array}{c}5,000- \\
6,000\end{array}$ & - & 6,242 & 194 & 97.0 & [57] \\
\hline & 11,783 & 13 & 13.5 & 2,740 & $143-160$ & - & - & 86.0 & [59] \\
\hline & - & 17.0 & - & - & - & - & - & 99.0 & {$[6]$} \\
\hline & - & $22-24$ & 16.8 & - & - & - & - & $96-98$ & [56] \\
\hline & - & 23.6 & - & - & - & - & - & 98.0 & {$[6]$} \\
\hline & - & 24.0 & - & - & - & - & - & 99.0 & [6] \\
\hline & - & 35.0 & - & - & - & - & - & 99.0 & {$[6]$} \\
\hline & 5,074 & 35.3 & 27 & - & - & 58,900 & 50.0 & $>99.9$ & [55] \\
\hline & - & 46 & 28.8 & 3,100 & - & 1,200 & 24.0 & 98.0 & [7] \\
\hline & - & 52 & 326 & 4,000 & - & 2,448 & 20.0 & 99.2 & [7] \\
\hline \multirow[t]{20}{*}{ Diclofenac } & - & 1.0 & - & - & - & - & - & 0.0 & [6] \\
\hline & - & 2 & 1.9 & 4,000 & - & 1,400 & 1,300 & 7.1 & [7] \\
\hline & 3,967 & 3 & 10 & 3,030 & $113^{*}$ & - & - & 28.0 & [26] \\
\hline & 105,300 & 5 & 16 & 2,450 & $127 *$ & - & - & 22.0 & [26] \\
\hline & - & 5.0 & - & - & - & - & - & 36.0 & {$[6]$} \\
\hline & 5,506 & 5.5 & 15 & 2,836 & $205^{*}$ & - & - & -88.0 & [26] \\
\hline & 15,300 & 5.5 & 15 & 1,743 & $154^{*}$ & - & - & -103 & [26] \\
\hline & 19,260 & 6 & 22 & 2,084 & $128^{*}$ & - & - & 30.0 & [26] \\
\hline & 20,000 & $6-10$ & 35 & - & $510-680$ & 100 & 485 & -79.4 & [62] \\
\hline & - & 9.6 & - & - & - & - & - & 9.0 & [6] \\
\hline & 17,994 & 10.5 & 13 & 2,105 & $51 *$ & - & - & -143.0 & [26] \\
\hline & - & $10-12$ & 7.3 & - & - & - & - & $22-33$ & [56] \\
\hline & - & 17.0 & - & - & - & - & - & 13.0 & [6] \\
\hline & - & $22-24$ & 16.8 & - & - & - & - & $30-34$ & [56] \\
\hline & - & 23.6 & - & - & - & - & - & 13.0 & [6] \\
\hline & - & 24.0 & - & - & - & - & - & 52.0 & [6] \\
\hline & 4,554 & 30 & 23 & 4,554 & $178^{*}$ & - & - & 77.0 & [26] \\
\hline & - & 35.0 & - & - & - & - & - & 3.0 & [6] \\
\hline & - & 46 & 28.8 & 3,100 & - & 905 & 780 & 13.8 & [7] \\
\hline & - & 52 & 326 & 4,000 & - & 3,190 & 1,680 & 47.3 & [7] \\
\hline
\end{tabular}


Table 4 (continued)

\begin{tabular}{|c|c|c|c|c|c|c|c|c|c|}
\hline Drug & $\begin{array}{c}\text { Flow rate / } \\
\mathbf{m}^{3} \mathbf{d}^{-1}\end{array}$ & SRT/ $d^{-1}$ & $\underset{1}{\text { HRT }} / \mathrm{h}^{-}$ & $\begin{array}{c}\text { MLSS / } \\
\mathrm{mg} \mathrm{l}^{-1} \\
\end{array}$ & $\begin{array}{l}\text { COD / } \\
\mathrm{mg} \mathrm{l}^{-1}\end{array}$ & $\begin{array}{l}\text { SS conc. } \\
/ \mathrm{ng} \mathrm{l}^{-1}\end{array}$ & $\begin{array}{c}\text { SE conc. } \\
/ \mathrm{ng} \mathrm{l}^{-1}\end{array}$ & $\begin{array}{c}\text { Removal } \\
1 \%\end{array}$ & Ref. \\
\hline \multirow[t]{25}{*}{ Carbamazepine } & - & 0.3 & - & - & - & - & - & 0.0 & {$[6]$} \\
\hline & 17,364 & 0.9 & 12 & - & - & 1,300 & 800 & 38.5 & [55] \\
\hline & - & 1.0 & - & - & - & - & - & -9.0 & [6] \\
\hline & 626,000 & 1.9 & 7 & - & - & 800 & 700 & 12.5 & [55] \\
\hline & - & 2 & 1.9 & 4,000 & - & 670 & 690 & -3.0 & [7] \\
\hline & 47,860 & 2.2 & 13 & - & - & 1,100 & 2,300 & -109.1 & [55] \\
\hline & 185,000 & 2.7 & 12 & - & - & 1,200 & 1,700 & -41.7 & [55] \\
\hline & 585,667 & 2.7 & 14 & - & - & 600 & 800 & -33.3 & [55] \\
\hline & 645,000 & 3.8 & 8.6 & - & - & 55.9 & 32.1 & 42.6 & [58] \\
\hline & 6,243 & 4.1 & 14 & - & - & 1,000 & 500 & 50.0 & [55] \\
\hline & 409,000 & 4.6 & 8.0 & - & - & 43.1 & 46.9 & -8.6 & [58] \\
\hline & 125,248 & 4.7 & 13 & - & - & 700 & 800 & -14.3 & [55] \\
\hline & $1,199,000$ & 5.0 & 7.1 & - & - & 50.5 & 45.4 & 10.2 & [58] \\
\hline & - & 5.0 & - & - & - & - & - & 6.0 & [6] \\
\hline & 210,000 & 8.4 & 8.9 & - & - & 173 & 117 & 32.4 & [58] \\
\hline & 68,498 & 9.6 & 15 & - & - & 700 & 700 & 0.0 & [55] \\
\hline & - & 9.6 & - & - & - & - & - & 9.0 & [6] \\
\hline & - & $10-12$ & 7.3 & - & - & - & - & $0-18$ & [56] \\
\hline & 366,898 & 12.6 & 23 & - & - & 900 & 1,700 & -88.9 & [55] \\
\hline & - & 17.0 & - & - & - & - & - & -11.0 & [6] \\
\hline & - & $22-24$ & 16.8 & - & - & - & - & $0-5$ & [56] \\
\hline & - & 35.0 & - & - & - & - & - & 1.0 & [6] \\
\hline & 5,074 & 35.3 & 27 & - & - & 1,000 & 900 & 10.0 & [55] \\
\hline & - & 46 & 28.8 & 3,100 & - & 325 & 426 & -31.1 & [7] \\
\hline & - & 52 & 326 & 4,000 & - & 704 & 952 & -26.1 & [7] \\
\hline
\end{tabular}

SRT, solids retention time; HRT, hydraulic retention time; MLSS, mixed liquor suspended solids;

COD, chemical oxygen demand; SS, settled sewage; SE, secondary effluent

*Biological oxygen demand

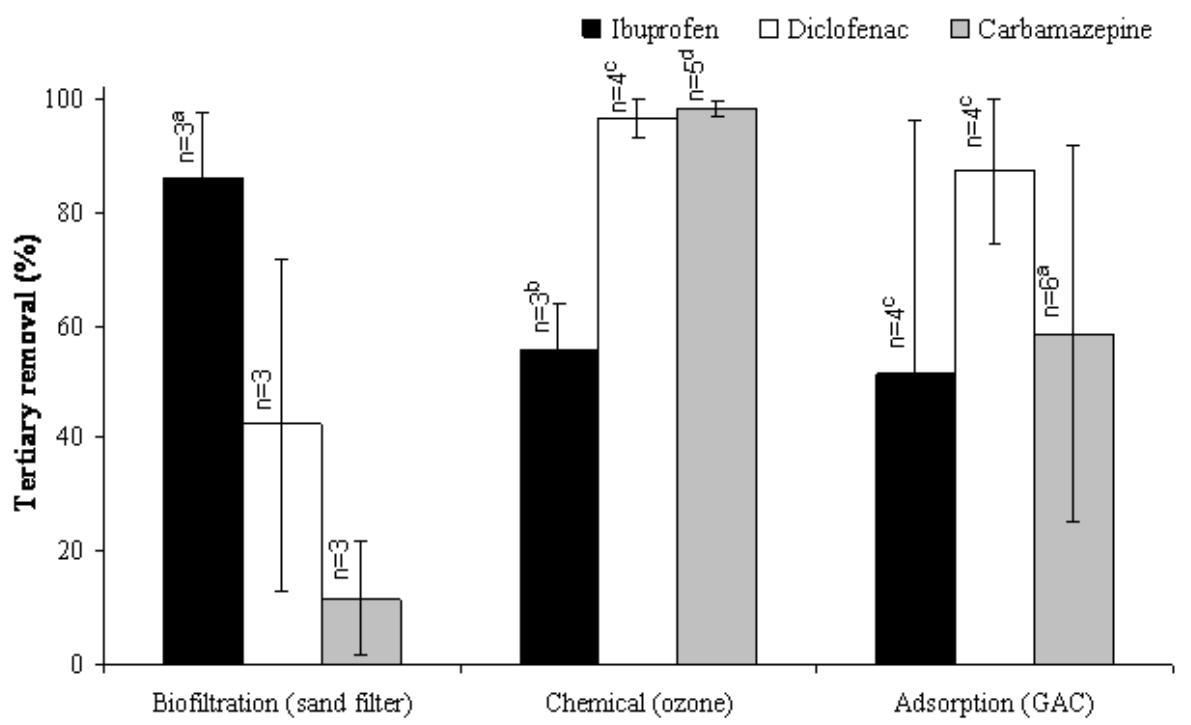

Figure 2. Removals of ibuprofen, diclofenac and carbamazepine reported in the literature for biofiltration, chemical and adsorption processes. Ibuprofen, diclofenac and carbamazepine represent drugs reasonably amenable to sorption and biodegradation, sorption only and neither sorption or biodegradation, respectively (removal data obtained from: [64-75] - Table 5, 6 and 7) ${ }^{\mathrm{a}} 1 / 3$ removed $<\mathrm{MQL}^{\mathrm{b}} 2 / 3$ removed $<\mathrm{MQL}^{\mathrm{c}} 3 / 4$ removed $<\mathrm{MQL}^{\mathrm{d}} 4 / 5$ removed $<\mathrm{MQL}$ 
Table 5. Removal of drugs from environmental waters by biofiltration processes

\begin{tabular}{|c|c|c|c|c|c|c|c|c|c|}
\hline Drug & $\begin{array}{l}\text { Proce } \\
\text { SS }\end{array}$ & Temp. $_{\text {C }}^{\circ}$ & $\underset{\mathbf{h}^{-1}}{\text { HRT }}$ & $\begin{array}{l}\text { Wastewate } \\
\text { r type }\end{array}$ & $\begin{array}{l}\text { Upfront } \\
\text { process }\end{array}$ & $\begin{array}{c}\text { SE. } \\
\text { conc. / } \\
\text { ng l }^{-1}\end{array}$ & $\begin{array}{c}\mathrm{TE} \\
\text { conc. / } \\
\text { ng l }^{-1}\end{array}$ & $\begin{array}{c}\text { Removal } \\
/ \%\end{array}$ & Ref. \\
\hline \multirow[t]{3}{*}{ Ibuprofen } & SF & - & $4-6$ & Municipal & - & $11,700^{\mathrm{a}}$ & 1,170 & 90 & [67] \\
\hline & SF & - & 0.3 & River & - & 276 & $<14$ & $>95$ & [64] \\
\hline & SF & - & 1 & Municipal & ASP & - & - & $73^{\mathrm{b}}$ & [68] \\
\hline \multirow[t]{3}{*}{ Diclofenac } & SF & - & $4-6$ & Municipal & - & $820^{\mathrm{a}}$ & 197 & 76 & [67] \\
\hline & SF & - & 0.3 & River & - & 252 & 181 & 28 & [64] \\
\hline & SF & 22 & 2 & Municipal & - & - & - & 23 & [73] \\
\hline \multirow[t]{3}{*}{$\mathrm{e}$} & SF & - & $4-6$ & Municipal & - & $2,060^{\mathrm{a}}$ & 1,833 & 11 & [67] \\
\hline & SF & - & 0.3 & River & - & 85 & 84 & 2 & [64] \\
\hline & SF & - & 1 & Municipal & ASP & - & - & $22^{\mathrm{b}}$ & [68] \\
\hline \multirow[t]{3}{*}{ Naproxen } & SF & - & $4-6$ & Municipal & - & $1,570^{\mathrm{a}}$ & 314 & 80 & [67] \\
\hline & $\mathrm{SF}$ & - & 0.3 & River & - & 170 & 24 & 86 & [64] \\
\hline & SF & - & 1 & Municipal & ASP & - & - & $32^{\mathrm{b}}$ & [68] \\
\hline Ketoprofen & SF & - & 1 & Municipal & ASP & - & - & $16^{\mathrm{b}}$ & [68] \\
\hline \multirow[t]{5}{*}{ EE2 } & SF & - & 0.3 & River & - & 316 & 246 & 22 & [64] \\
\hline & SF & 18 & - & Municipal & ASP & - & 0.2 & 82 & [76] \\
\hline & $\mathrm{SF}$ & - & - & Municipal & ASP & - & - & 7 & [77] \\
\hline & SF & - & - & Municipal & OD & - & - & 9 & [77] \\
\hline & SF & $22-25$ & - & Municipal & - & 109 & 64 & 41 & [78] \\
\hline \multirow[t]{2}{*}{ Erythromycin } & $\mathrm{SF}$ & - & 0.3 & River & - & 104 & 75 & 28 & [64] \\
\hline & SF & $19-22$ & 0.4 & Municipal & ASP & - & - & 20 & [79] \\
\hline
\end{tabular}

comprised of a diverse community of micro-organisms embedded within a matrix of extracellular polymeric substances (EPS) consisting of proteins, nucleic acids, polysaccharides and amphiphilic polymeric compounds [81]. The composition of EPS shifts with biofilm age [81], and is known to influence EE2 uptake [54]. The high tendency of some drugs to partition to solid organic matter, similar to biofilms has been confirmed by ASE followed by LC-MS/MS analysis [30, 38-40]. Those hydrophobic drugs with a comparatively high $\log \mathrm{K}_{\mathrm{ow}}(>4)$ (e.g., diclofenac and fluoxetine) are considered to have a tendancy to sorb to solid organic surfaces such as biofilms [82-83]. However, sorption cannot be predicted by hydrophobicity alone [83-84]; other properties such as molecular weight and ionic speciation are of known importance [83], as is the nature of other dissolved species with which they may interact [85]. Extracellular polymeric substances offer both anionic and cationic functional groups for the exchange of charged species [83]. At a $\mathrm{pH}$ typical of municipal wastewaters (e.g., 7-8), EPS is negatively charged [83] with pKa's generally ranging from 6.2 to 10.1 [86]. Those drugs whose pKa is $<7$ (e.g., ibuprofen, diclofenac, 


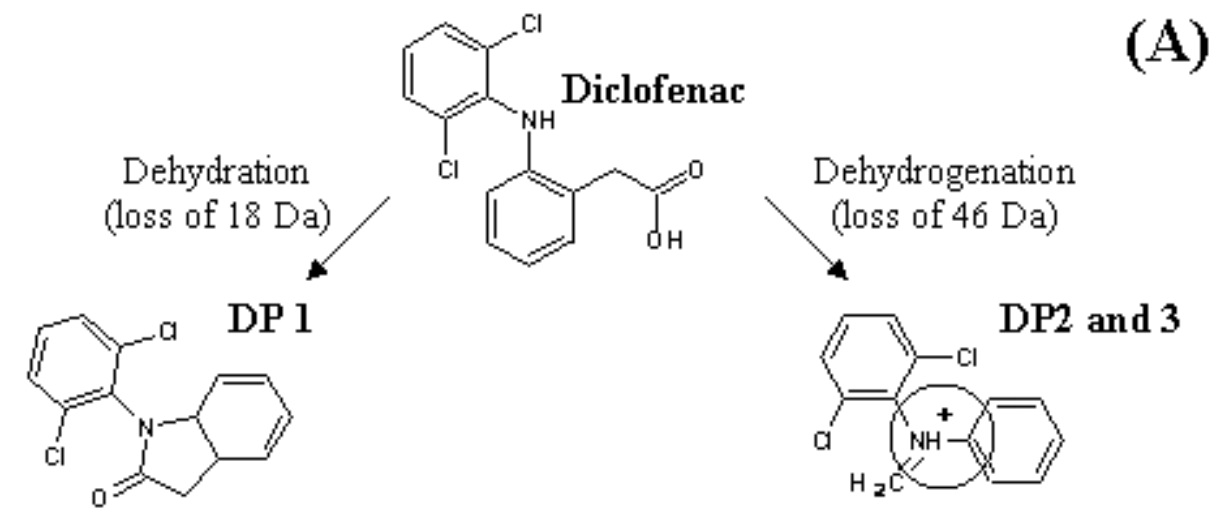

Structure confirmed by reference standard Isomers with position of $-\mathrm{CH}_{2}$ not defined
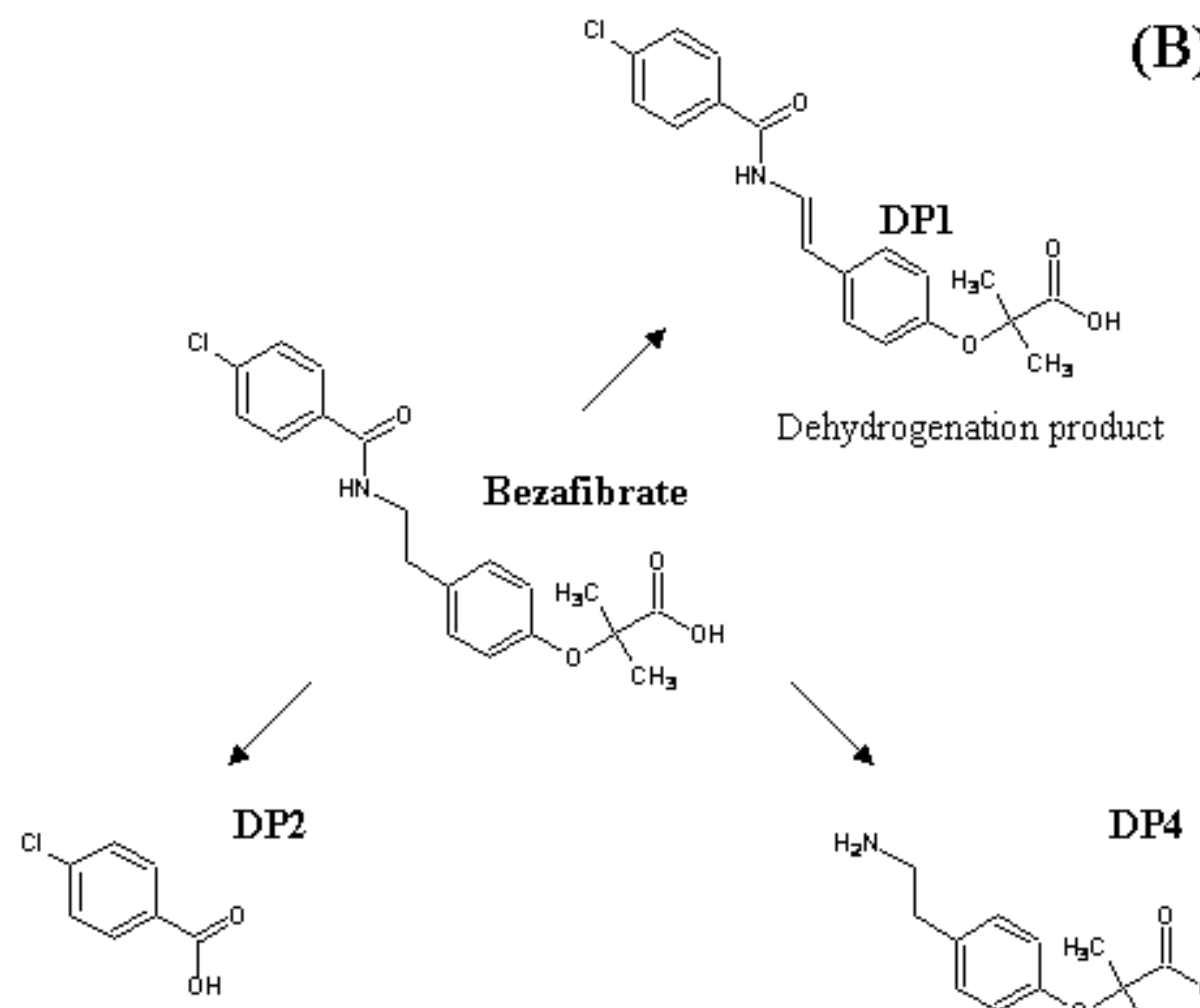

Structure confirmed by reference standard<smiles>O=C(O)c1ccc(O)cc1</smiles>

Structure confirmed by reference standard<smiles>CC(C)(Oc1ccc(CCN)cc1)C(=O)O</smiles><smiles>CC(C)(Oc1ccc(CCN)c(O)c1)C(=O)O</smiles>

Positions of $-\mathrm{OH}$ not defined

Figure 3. Biological degradation products (DP) of diclofenac (A, adapted from [90]) and the proposed biotransformation pathway of bezafibrate ( $B$, adapted from [50]) 
naproxen, ketoprofen and oxytetracyline) (Table 1) will themselves be negatively charged and repulsion with the biofilm may restrict sorption. Removal will also be influenced by biofilm porosity. Drugs whose molecular size is comparatively large (e.g., erythromycin), will have a reduced rate of mass transfer between the liquid medium and the biofilm, limiting partitioning [83]. Drugs which are comparatively smaller $\left(<300 \mathrm{~g} \mathrm{~mol}^{-1}\right)$ and relatively hydrophobic in nature ( $\log \mathrm{K}_{\text {ow }}$ 's $>3$ ) (e.g., ibuprofen, diclofenac, naproxen, ketoprofen, propranolol and EE2, Table 1) are expected to partition well within the biofilm matrix.

Sorption is also considered to be an intermediate step in biodegradation [52, 54]. Assuming similar behaviour to EE2 and other hazardous chemicals in biological processes, drug biodegradation is likely to be mediated on the surface and/or within intact bacterial cells [52, 54, 87]. Free ammonia mono-oxygenase enzymes released by lysis extracellularly are not likely to be involved in biodegradation [52, 87]. Gaulke et al [88] observed that EE2 removal in nitrifying batch studies was not by nitrifying bacteria activity, dismissing the hypothesis that nitrification augments EE2 removals [89]. The synthesis of nitro-EE2 confirmed that EE2 is nitrated at high ammonia feed concentrations caused by the high production of nitrates; EE2 removal here is an artefact of laboratory scale investigation. Biodegradation at environmentally representative conditions is by heterotrophic micro-organisms, capable of scavenging a broad range of organic material [87]. Interestingly, differences in biodegradation are observed for drugs which sorb similarly to biomass (e.g., ibuprofen and diclofenac, Figure 1) suggesting chemical structure controls susceptibility to biological attack. The structure of ibuprofen is comparatively simpler than that of diclofenac (i.e., single aromatic ring and non-halogenated, Table 2) which may aid its biodegradation. Drugs of increasing structural complexity and elemental diversity such as antibiotics (e.g., ofloxacin, erythromycin and oxytetracycline) may be less favourable to biodegradation especially considering their possible toxicity to bacteria. However, 'biodegradation' here is not indicative of complete mineralisation. Drugs rich in functional groups provide more possible sites for biological attack, inducing a change to the parent structure. Kosjek et al [90] investigated the degradation products of diclofenac, utilising Oasis HLB SPE and UPLC separations to ensure adequate sensitivity required by QqTOF. In full scan mode the total ion chromatogram (TIC) was screened and a protonated compound was then selected for further product ion scans [90]. Accurate mass measurements and in-source fragmentation enabled chemical structure elucidation of three biotransformation products (Figure 3A). Similarly Helbing et al [50] used LIT-Orbitrap MS to identify five degradation products of bezafibrate (Figure 3B). The dehydrogenation product (DP1) is structurally similar to the parent drug indicating it may behave similarly in the environment. 


\subsection{Chemical oxidation}

Titanium dioxide photocatalysis [91] and Fenton chemistry (i.e., catalytic oxidation of hydrogen peroxide) [92] have been applied to water treatment however ozone is the most well established and studied of the chemical processes for drug removal. Ozone treatment enhances the removal of all drugs including carbamazepine where removals of $\geq 96 \%$ are observed [65, 70-71, 73-74] (Figure 2, Table 6). However, typical ozone doses applied during water treatment often do not enable full mineralization of drugs [93], likely to be caused by the clouding influence of the matrix. Wastewaters contain relatively high concentrations of bulk organics which can shield targeted chemicals from removal, quenching the ozone dose. Furthermore, Huber et al [93] observed that following removal of EE2 from clean water by a high ozone dose, a slow re-appearance of the drug $(0.1-0.5 \%$ of the initial concentration) occurred. It is hypothesized that some EE2 is in the form of hydroperoxides which are not readily reactive to ozone. This could be greater in wastewater where clouding will reduce reaction kinetics and this will hinder the complete mineralisation of the parent drug. Degradation by ozonation can occur selectively by direct ozone attack itself and nonselectively by hydroxyl radicals formed upon ozone decay [93]. Ozone reacts rapidly with phenols at neutral or basic $\mathrm{pH}$ [94] therefore it will readily attack the phenolate anion of EE2 and oxytetracycline. It also selectively attacks amines and double bonds of aliphatic chemicals [95]. The chemical structure of all the drugs considered here (except ibuprofen) are highly susceptible to direct ozone attack (Table 2). Hydroxyl radicals are less selective and react with a range of chemical functional groups. The non-selective behaviour of the hydroxyl radicals can induce complex reaction pathways [93]. Numerous authors observed high removals of various drugs by ozone treatment, often to concentrations below their MQLs [65, 70-71]. However, complete removal of the parent drug does not necessarily represent removal of toxicity. Structurally similar degradation products of potential toxicity can be formed and remain undetected using conventional MS/MS (i.e., QqQ). A large number of degradation products for various drugs have been observed $[93,96]$. Non-target screening of ozone treated water enabled determination of 17 degradation products of diclofenac [96]

(Figure 4). The majority of these products are structurally similar to the parent drug indicating similar behaviour in the receiving environment. Again these were identified by Oasis HLB extraction and QqTOF detection. Accurate mass spectra were collected at mass to charge $(\mathrm{m} / \mathrm{z})$ ratios $>50$ encompassing all degradation products of notable size.

\subsection{Adsorption by activated carbon}

Activated carbon often contained in a packed bed or filter is a highly porous medium offering a large internal surface area for sorption to take place. Performance is dependant on activated carbon properties (e.g., pore size, surface charge) and solute characteristics (e.g., shape, size) 
Table 6. Removal of drugs from environmental waters by ozone treatment

\begin{tabular}{|c|c|c|c|c|c|c|c|c|c|}
\hline Drug & Process & $\begin{array}{c}\text { Chemical } \\
\text { dose }\end{array}$ & $\underset{\mathbf{h}^{-1}}{\text { HRT }}$ & $\begin{array}{c}\text { Wastewater } \\
\text { type }\end{array}$ & $\begin{array}{l}\text { Upfront } \\
\text { process }\end{array}$ & $\begin{array}{c}\text { SE. } \\
\text { conc. / } \\
\text { ng l-1 }\end{array}$ & $\begin{array}{c}\text { TE } \\
\text { conc. / } \\
\text { ng I }\end{array}$ & $\begin{array}{c}\text { Remova } \\
1 / \%\end{array}$ & Ref. \\
\hline \multirow[t]{3}{*}{ Ibuprofen } & Ozone & $\begin{array}{c}10-15 \mathrm{mg} \\
1^{-1}\end{array}$ & 0.3 & Municipal & ASP & 130 & $<50$ & $>62$ & [65] \\
\hline & Ozone & $2 \mathrm{mg} \mathrm{l}^{-1}$ & 0.2 & $\begin{array}{l}\text { Surface } \\
\text { waters }\end{array}$ & - & - & - & $40-77$ & [75] \\
\hline & Ozone & $3 \mathrm{mg} \mathrm{l}^{-1}$ & 0.5 & Municipal & $\begin{array}{c}\mathrm{ASP}+ \\
\mathrm{SF}\end{array}$ & - & - & $>46^{\mathrm{a}}$ & {$[68]$} \\
\hline \multirow[t]{4}{*}{ Diclofenac } & Ozone & $\begin{array}{c}0.6 \mathrm{~g} \mathrm{O}_{3} \mathrm{~g} \\
\mathrm{DOC}_{0}^{-1}\end{array}$ & - & Municipal & ASP & 2,000 & $<10$ & $>99$ & [71] \\
\hline & Ozone & $3 \mathrm{mg} \mathrm{l}^{-1}$ & 0.3 & Municipal & - & - & - & 92 & [73] \\
\hline & Ozone & $50 \mu \mathrm{M}$ & $<0.1$ & Municipal & - & 433 & $<1$ & $>99$ & {$[70]$} \\
\hline & Ozone & $\begin{array}{c}10-15 \mathrm{mg} \\
1^{-1} \\
\end{array}$ & 0.3 & Municipal & ASP & 1,300 & $<50$ & $>96$ & [65] \\
\hline \multirow[t]{5}{*}{$\begin{array}{c}\text { Carbamazep } \\
\text { ine }\end{array}$} & Ozone & $\begin{array}{c}0.6 \mathrm{~g} \mathrm{O}_{3} \mathrm{~g} \\
\mathrm{DOC}_{0}^{-1}\end{array}$ & - & Municipal & ASP & 900 & $<1$ & $>99$ & [71] \\
\hline & Ozone & $3 \mathrm{mg} \mathrm{l}^{-1}$ & 0.3 & Municipal & - & - & - & 96 & [73] \\
\hline & Ozone & $130 \mu \mathrm{M}$ & 0.1 & Municipal & - & 106 & $<1$ & $>99$ & {$[70]$} \\
\hline & Ozone & $1 \mathrm{mg} \mathrm{l}^{-1}$ & - & Municipal & GAC & 67 & 1 & 99 & [74] \\
\hline & Ozone & $\begin{array}{c}10-15 \mathrm{mg} \\
\mathrm{l}^{-1}\end{array}$ & 0.3 & Municipal & ASP & 2,100 & $<50$ & $>98$ & {$[65]$} \\
\hline \multirow[t]{2}{*}{ Naproxen } & Ozone & $3 \mathrm{mg} \mathrm{l}^{-1}$ & 0.5 & Municipal & $\begin{array}{c}\mathrm{ASP}+ \\
\mathrm{SF}\end{array}$ & - & - & $>99^{a}$ & {$[68]$} \\
\hline & Ozone & $\begin{array}{c}10-15 \mathrm{mg} \\
1^{-1}\end{array}$ & 0.3 & Municipal & ASP & 100 & $<50$ & $>50$ & {$[65]$} \\
\hline \multirow[t]{3}{*}{ Bezafibrate } & Ozone & $\begin{array}{c}0.6 \mathrm{~g} \mathrm{O}_{3} \mathrm{~g} \\
\mathrm{DOC}_{0}^{-1}\end{array}$ & - & Municipal & ASP & 1,500 & 345 & 77 & [71] \\
\hline & Ozone & $340 \mu \mathrm{M}$ & 0.3 & Municipal & - & 115 & 4 & 97 & {$[70]$} \\
\hline & Ozone & $2 \mathrm{mg} \mathrm{l}^{-1}$ & 0.2 & $\begin{array}{c}\text { Surface } \\
\text { waters }\end{array}$ & - & - & - & $>98$ & [75] \\
\hline Fluoxetine & Ozone & $50 \mu \mathrm{M}$ & $<0.1$ & Municipal & - & 17 & $<2$ & $>88$ & [70] \\
\hline Ketoprofen & Ozone & $3 \mathrm{mg} \mathrm{l}^{-1}$ & 0.5 & Municipal & $\begin{array}{c}\mathrm{ASP}+ \\
\mathrm{SF}\end{array}$ & - & - & $73^{\mathrm{a}}$ & {$[68]$} \\
\hline
\end{tabular}

[69]. Preferential attraction to the activated carbon surface is by hydrogen bonding and

London forces creating a strong binding affinity. Even moderately hydrophobic chemicals

$\left(\log \mathrm{K}_{\mathrm{ow}}>2\right.$ ) have a high propensity to removal [95]. The availability of some drugs as anions causes them to be attracted to the carbon surface. As a result substantial removals of hydrophobic and hydrophilic drugs have been observed by granular activated carbon (GAC) [66, 73-74] (Figure 2, Table 7). Carbamazepine removals up to $97 \%$ have been achieved [73]. Despite the very hydrophilic nature of oxytetracycline (Table 1), its large molecular size is likely to be entrapped within the highly porous structure of the activated carbon. At fullscale treatment processes low removals have been observed for some drugs; ibuprofen (16\%) [69], carbamazepine (16-23\%) [69, 72] and propranolol (17\%) [72] (Table 7). The quality of the secondary effluent will have a significant influence on the performance of GAC through the competition for available sorption sites [69]. The frequency of replacement/regeneration of the activated carbon medium is another controlling factor to its success, especially whilst treating wastewaters comprising relatively high concentrations of 
bulk organics. This may account for large variations in drug removals observed between processes. Chiu et al [97] demonstrated the possibility of in situ catalytic regeneration of GAC using iron nano-catalysts. This could provide an effective means of regeneration in the future, limiting variations in performance currently observed.

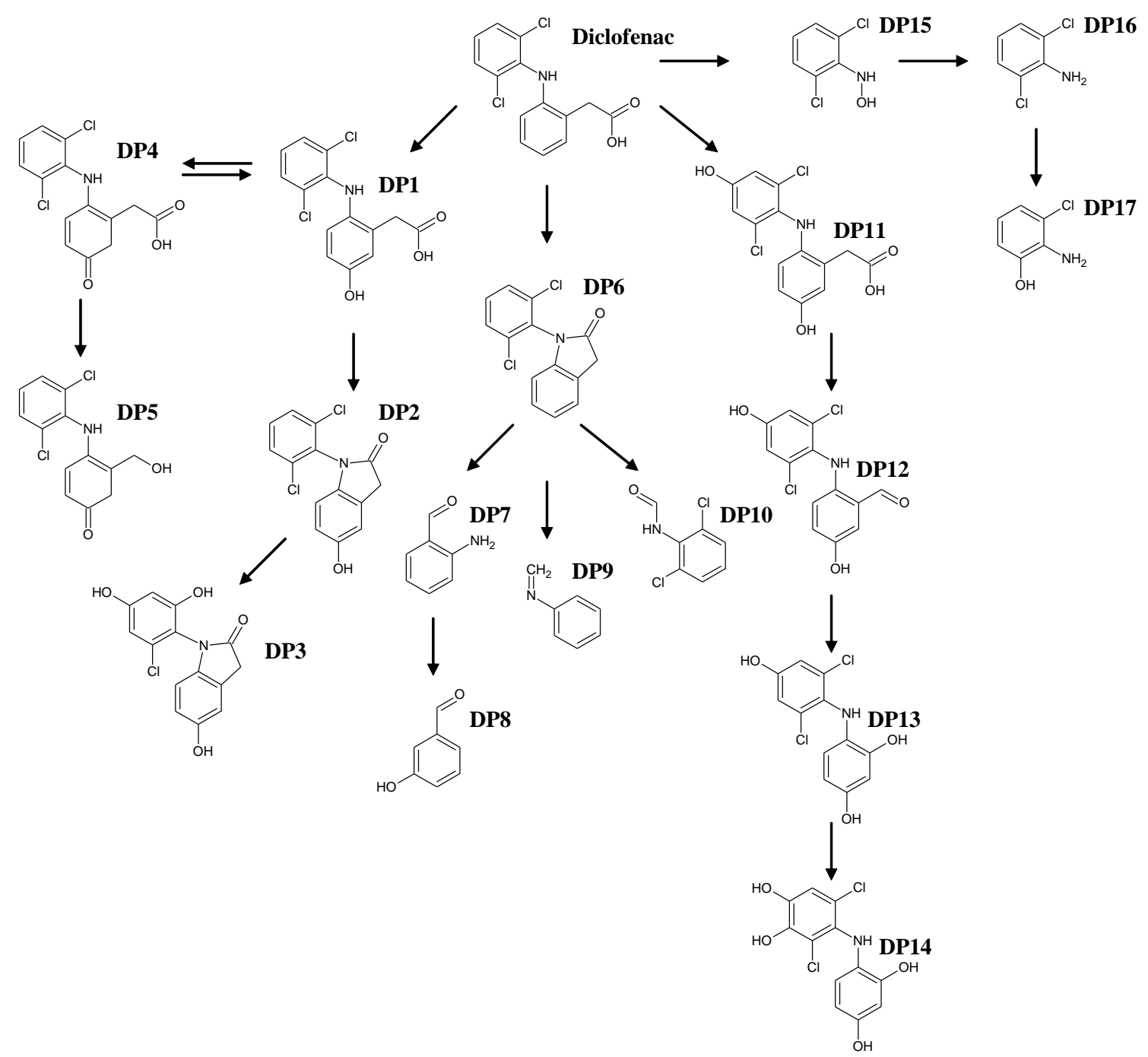

Figure 4. Degradation intermediates formed by ozone treatment of diclofenac and proposed degradation pathways (adapted from [96])

\section{Future trends}

Secondary effluents typically demand analytical MQLs in the low $n g \mathrm{l}^{-1}$ range to determine most drugs. Reported analytical methods are well suited for determining drugs in the aqueous phase of secondary effluents. To demonstrate, no data in Figure 1 (and Table 4) was reported below MQL ( $\mathrm{n}=80$ ). Despite proposed legislative targets being applied to the aqueous phase 
Table 7. Removal of drugs from environmental waters by adsorption processes

\begin{tabular}{|c|c|c|c|c|c|c|c|c|}
\hline Drug & Process & $\begin{array}{c}\text { HRT } \\
\mathbf{h}^{-1}\end{array}$ & $\begin{array}{c}\text { Wastewater } \\
\text { type }\end{array}$ & $\begin{array}{l}\text { Upfront } \\
\text { process }\end{array}$ & $\begin{array}{c}\text { SE. } \\
\text { conc./ } \\
\text { ng l }^{-1}\end{array}$ & $\begin{array}{c}\text { TE } \\
\text { conc./ } \\
\text { ng l }^{-1}\end{array}$ & $\begin{array}{c}\text { Removal } \\
/ \%\end{array}$ & Ref. \\
\hline \multirow[t]{4}{*}{ Ibuprofen } & GAC & 0.3 & Municipal & ASP & 64 & $<10$ & $>84$ & [74] \\
\hline & GAC & - & Raw water & - & 23 & $<1$ & $>96$ & [66] \\
\hline & GAC & - & Surface water & - & 1.1 & $<1$ & $>10$ & [69] \\
\hline & GAC & - & - & SF & 8,760 & 7,325 & 16 & [69] \\
\hline \multirow[t]{4}{*}{ Diclofenac } & GAC & 0.3 & Municipal & ASP & 99 & $<10$ & $>90$ & [74] \\
\hline & GAC & - & Municipal & ASP & - & - & $>98$ & [72] \\
\hline & BAC & 2 & Municipal & MBR & - & - & 92 & [73] \\
\hline & GAC & - & - & $\mathrm{SF}$ & 3.2 & $<1$ & $>69$ & [69] \\
\hline \multirow[t]{6}{*}{ Carbamazepine } & GAC & 0.3 & Municipal & ASP & 250 & 67 & 73 & [74] \\
\hline & GAC & - & Municipal & ASP & - & - & 23 & [72] \\
\hline & BAC & 2 & Municipal & MBR & - & - & 97 & [73] \\
\hline & GAC & - & Raw water & - & 8 & $<1$ & $>87$ & [66] \\
\hline & GAC & - & Surface water & - & 2.2 & $<1$ & $>55$ & [69] \\
\hline & GAC & - & - & $\mathrm{SF}$ & 199 & 168 & 16 & [69] \\
\hline Propranolol & GAC & - & Municipal & ASP & - & - & 17 & [72] \\
\hline EE2 & GAC & - & Municipal & ASP & - & - & $>43$ & [72] \\
\hline \multirow[t]{2}{*}{ Erythromycin } & GAC & 0.3 & Municipal & ASP & 270 & 28 & 90 & [74] \\
\hline & BAC & 2 & Municipal & MBR & - & - & 92 & [73] \\
\hline
\end{tabular}

HRT, hydraulic retention time; SE, secondary effluent; TE, tertiary effluent; ASP, activated sludge plant; GAC, granular activated carbon; BAC, biologically activated carbon; MBR, membrane bioreactor

of wastewaters (i.e., a pre-filtered sample); suspended solids can provide a pathway to their release into the environment [30]. Their determination in the particulate phase is also essential for fate evaluation. Suspended solids are ubiquitous to wastewaters and can vary spatially and temporally. Particulate bound drugs often go undetermined (Table 3), owing to the complexity of the matrix and the additional analytical requirements it demands. The proposed requirement to undertake particulate phase analysis to determine drug fate is most pertinent to secondary processes which receive relatively high concentrations of suspended solids. The relatively hydrophobic nature of some drugs can cause them to partition well to solids. For example crude wastewaters can contain $>50 \%$ of fluoxetine bound to particulates [30]. Monitoring here enables complete process mass balances to be determined, aiding fate and performance understanding. Particulate fate understanding may indicate a clouding influence during treatment which limits removal. Activated sludge sorption and biodegradation may be restricted for drugs associated with particulates in the receiving wastewater. This could lead to conventional process optimisation to enhance drug removal. For example, the use of micro-screens in place of conventional primary sedimentation tanks 
could enhance particulates removal from the crude stream. However, there is a substantial gap between drug concentrations achieved by the current operations of existing secondary assets and proposed legislative targets [19] (Table 2, Table 4). Therefore there is an expectant need for tertiary treatment technologies to target these specific chemicals.

Tertiary treatment processes enhance drug removal, significantly reducing effluent concentrations. To fully ascertain tertiary process performance, analytical methods require MQLs $<10 \mathrm{ng} \mathrm{l}^{-1}[64,70-71,74]$, and ideally $<1 \mathrm{ng} \mathrm{l}^{-1}[66,69-71]$ (Tables A2-4). This poses a further analytical challenge as such concentrations cannot be ascertained for the majority of drugs with current MQLs. To illustrate, concentrations of the representative drugs; ibuprofen, diclofenac and carbamazepine in sand filtration, ozone and activated carbon treated effluents were reported below MQL in $49 \%$ of cases ( $n=35$ ) (Figure 2). Despite these being below proposed legislative targets for most drugs (Table 2), monitoring at these concentrations is needed as the cumulative toxicological effect of drugs is not known. This could result in a future reduction in legislative requirements. To illustrate, the previous EE2 predicted no effect concentration (PNEC) in the UK was $0.1 \mathrm{ng} \mathrm{l}^{-1}$ [98]. The proposed EQS is now 0.035 ng $1^{-1}$ following its classification as a priority hazardous chemical [12]. This has created a serious analytical burden as such concentrations are now beyond current analytical capabilities [23]. Lowering current MQLs is also needed to assess breakdown reaction completeness. The first stage of this is to determine parent drug removal. Further investigation of specific breakdown mechanisms to understand the criticality between parent drug final concentration, and degradation product production is needed. Methodologies to quantify the full range degradation products will be restricted in the short term by the lack of unique reference standards available for these. However, the identification of numerous degradation products in both biological and chemical processes has brought attention to their presence and created a demand for their commercial availability (Figures 3-4). The complimentary use of biological assays would improve understanding of the synergistic toxicological effect of multiple drugs and their degradation products at low concentration. Process design and operation must integrate the removal of these intermediates which can be of greater concern than the parent chemical due to their subsequent transformation to more toxic chemicals in the environment [47].

Lowering drug MQLs requires existing analytical method optimisation. The low recoveries $(<50 \%)$ typically stipulated prior to internal standard correction [25], can be improved to reduce the achievable MQL. Baker and Kasprzyk-Hodern [99] gave an excellent account of sample preparations parameters which can influence recovery. For example drugs can be adsorbed onto glassware surfaces during handling and processing. Using silanized SPE 
extract vials gave recoveries six times higher than non-silanized vials for some drugs. All glassware used during sample collection and processing requires silanization to ensure maximum recoveries. Silanization of glassware is not mentioned in the procedures of most reported analytical methods [17-18, 25, 33]. Improving chromatographic separations could also significantly increase detection capabilities. Incorporating a large number of drugs into a single short UPLC run $(<10$ minutes) results in a number of co-eluting peaks [17-18, 25]. Despite the use of mass scanning windows which typically range from 0.3 to 2 minutes in length for UPLC separations [17-18], sensitivity can be lost whilst simultaneously scanning for a number of transitions registered at the same time [17]. To demonstrate, Gros et al [18] reports up to 10 drugs co-eluting within a 0.1 minute time period with scanning windows of 0.5 minutes. Thus, only monitoring for one drug (of most criticality) in this time period could increase sensitivity and notably reduce the MQL.

Tertiary processes receive secondary effluents comprising comparatively high concentrations of dissolved organics (e.g., colloids). However, knowledge of drug behaviour in the charged colloidal fraction of wastewater is limited. Shen et al [100] successfully showed humic acid, a small molecular weight charged species could effectively retain the hormone, estrone in solution. This can restrict sorption in tertiary processes characterised by very short contact times. Furthermore, the complexity of the colloidal fraction and its interaction with the drugs could also lead to incomplete breakdown reactions in both biological and chemical processes. A fractionation step during sample pre-treatment to separate dissolved colloids by molecular weight will aid this. It is postulated that drugs will preferentially be in specific size fractions. This is likely to vary between drugs due to the range of physicochemical behaviour they exhibit. Understanding drug fate in the colloidal fraction of wastewater is essential for tertiary process selection, diagnosis and optimisation.

\section{Conclusion}

Advances in both quantitative and qualitative determinations of pharmaceutical drugs have aided the understanding of their occurrence and fate during wastewater treatment. A robust understanding of tertiary process performance is now needed by improving analytical focus. An appropriate treatment strategy could then be implemented to ensure adequate protection of the aquatic environment is achieved.

\section{Acknowledgements}

B.P. is grateful to the U.K. Engineering and Physical Sciences Research Council (EPSRC) for the award of a PhD scholarship. We would like to thank the following UK Water Companies: 
Northumbrian Water, Anglian Water, Severn Trent Water, Yorkshire Water and United

Utilities for providing their support and funding.

\section{References}

[1] N.D. Bedding, A.E. McIntyre, R. Perry, J.N. Lester, Organic contaminants in the aquatic environment. I. Sources and occurrence, Sci. Total Environ. 25 (1982) 143-167.

[2] R.S.K. Buisson, P.W.W. Kirk, J.N. Lester, Determination of chlorinated phenols in water, wastewater, and wastewater sludge by capillary GC/ECD, Chromatogr. Sci., 22 (1984) 339342.

[3] A.M. Robertson, J.N. Lester, Supercritical fluid extraction of s-triazines and phenylurea herbicides from sediment, Environ. Sci. Technol., 28 (1994) 346-351.

[4] O.A.H. Jones, N. Voulvoulis, J.N. Lester, Human pharmaceuticals in the aquatic environment: a review, Environ. Technol., 22 (2001) 1383-1394.

[5] O.A.H. Jones, N. Voulvoulis, J.N. Lester, Potential impact of pharmaceuticals on environmental health, Bull. World Health Org., 81 (2003) 768-769.

[6] N. Kreuzinger, M. Clara, B. Strenn, H. Kroiss, Relevance of the sludge retention time (SRT) as design criteria for wastewater treatment plants for the removal of endocrine disruptors and pharmaceuticals from wastewater, Water Sci.Technol. 50 (2004) 149-156.

[7] M. Clara, N. Kreuzinger, B. Strenn, O. Gans, H. Kroiss, The solids retention time - A suitable design parameter to evaluate the capacity of wastewater treatment plants to remove micropollutants, Water Res. 39 (2005) 97-106.

[8] R.L. Gomes, M.D. Scrimshaw, J.N. Lester, Determination of endocrine disrupters in sewage treatment and receiving waters, Trends Anal. Chem., 22 (2003) 697-707.

[9] R.L. Gomes, E. Avcioglu, M.D. Scrimshaw, J.N. Lester, Steroid estrogen determination in sediment and sewage sludge: a critique of chromatographic/mass spectrometry methods incorporating a case study in method development, Trends Anal. Chem. 23 (2004) 737-744.

[10] R.L. Gomes, H.E. Deacon, K.M. Lai, J.W. Birkett, M.D. Scrimshaw, J.N. Lester, An assessment of the bioaccumulation of estrone in Daphnia magna, Environ. Toxicol. Chem., 23 (2004) 105-108.

[11] European Commission. Priority Substances Daughter Directive-Directive 2008/105/EC of the European Parliament and of the Council of 16 December 2008 on environmental quality standards in the field of water policy. 2008.

[12] European Commission. Proposal for a Directive of the European Parliament and of the council amending Directives 2000/60/EC and 2008/105/EC as regards priority substances in the field of water policy. 2012. 
[13] J.P. Sumpter, S. Jobling, The occurrence, causes, and consequences of estrogens in the aquatic environment, Environ. Toxicol. Chem. 32 (2013) 249-251.

[14] M. Galus, J. Jeyaranjaan, E. Smith, H. Li, C. Metcalfe, J.Y. Wilson, Chronic effects of exposure to a pharmaceutical mixture and municipal wastewater in zebrafish, Aquatic Toxicol. 132-133 (2013) 212-222.

[15] T. Brodin, J. Fick, M. Jonsson, J. Klaminder, Dilute concentrations of a psychiatric drug alter behavior of fish from natural populations, Science. 339 (2013) 814-815.

[16] EPI Suite. http://www.epa.gov/oppt/exposure/pubs/episuite.htm (accessed 07.01.13).

[17] R. López-Serna, M. Petrović, D. Barceló, Development of a fast instrumental method for the analysis of pharmaceuticals in environmental and wastewaters based on ultra high performance liquid chromatography (UHPLC)-tandem mass spectrometry (MS/MS), Chemosphere. 85 (2011) 1390-1399.

[18] M. Gros, S. Rodríguez-Mozaz, D. Barceló, Fast and comprehensive multi-residue analysis of a broad range of human and veterinary pharmaceuticals and some of their metabolites in surface and treated waters by ultra-high-performance liquid chromatography coupled to quadrupole-linear ion trap tandem mass spectrometry, J. Chromatogr. A. 1248 (2012) 104-121.

[19] M. Gardner, S. Comber, M.D. Scrimshaw, E. Cartmell, J. Lester, B. Ellor, The significance of hazardous chemicals in wastewater treatment works effluents, Sci. Total Environ. 437 (2012) 363-372.

[20] B. Kasprzyk-Hordern, R.M. Dinsdale, A.J. Guwy, The removal of pharmaceuticals, personal care products, endocrine disruptors and illicit drugs during wastewater treatment and its impact on the quality of receiving waters, Water Res. 43 (2009) 363-380.

[21] C. Wu, J.D. Witter, A.L. Spongberg, K.P. Czajkowski, Occurrence of selected pharmaceuticals in an agricultural landscape, western Lake Erie basin, Water Res. 43 (2009) 3407-3416.

[22] I. Ferrer, E.M. Thurman, Analysis of 100 pharmaceuticals and their degradates in water samples by liquid chromatography/quadrupole time-of-flight mass spectrometry, J.

Chromatogr. A. 1259 (2012) 148-157.

[23] B. Petrie, E.J. McAdam, K.H. Richards, J.N. Lester, E. Cartmell, Application of ultraperformance liquid chromatography-tandem mass spectrometry for the determination of steroid oestrogens in wastewaters, Int. J. Environ. Anal. Chem.

DOI:10.1080/03067319.2012.717272 (2012)

[24] C. Hignite, D.L. Azarnoff, Drugs and drug metabolites as environmental contaminants: chlorophenoxyisobutyrate and salicylic acid in sewage water effluent, Life Sci. 20 (1977) 337-341.

[25] E. Gracia-Lor, J.V. Sancho, F. Hernández, Multi-class determination of around 50 pharmaceuticals, including 26 antibiotics, in environmental and wastewater samples by ultra- 
high performance liquid chromatography-tandem mass spectrometry, J. Chromatogr. A. 1218 (2011) 2264-2275.

[26] L. Lishman, S.A. Smyth, K. Sarafin, S. Kleywegt, J. Toito, T. Peart, B. Lee, M. Servos, M. Beland, P. Seto, Occurrence and reductions of pharmaceuticals and personal care products and estrogens by municipal wastewater treatment plants in Ontario, Canada., Sci. Total Environ. 367 (2006) 544-558.

[27] E.J. McAdam, J.P. Bagnall, A. Soares, Y.K.K. Koh, T.Y. Chiu, M.D. Scrimshaw, J.N. Lester, E. Cartmell, Fate of alkylphenolic compounds during activated sludge treatment: Impact of loading and organic composition, Environ. Sci. Technol. 45 (2011) 248-254.

[28] C. Ort, M.G. Lawrence, J. Reungoat, J.F. Mueller, Sampling for PPCPs in wastewater systems: Comparison of different sampling modes and optimization strategies, Environ. Sci. Technol. 44 (2010) 6289-6296.

[29] C. Ort, M.G. Lawrence, J. Rieckermann, A. Joss, Sampling for pharmaceuticals and personal care products (PPCPs) and illicit drugs in wastewater systems: Are your conclusions valid? A critical review, Environ. Sci. Technol. 44 (2010) 6024-6035.

[30] D.R. Baker, B. Kasprzyk-Hordern, Multi-residue determination of the sorption of illicit drugs and pharmaceuticals to wastewater suspended particulate matter using pressurised liquid extraction, solid phase extraction and liquid chromatography coupled with tandem mass spectrometry, J. Chromatogr. A. 1218 (2011) 7901-7913.

[31] M. Gros, M. Petrović, D. Barceló, Development of a multi-residue analytical methodology based on liquid chromatography-tandem mass spectrometry (LC-MS/MS) for screening and trace level determination of pharmaceuticals in surface and wastewaters, Talanta. 70 (2006) 678-690.

[32] J. Radjenović, M. Petrović, D. Barceló, M. Petrović, Advanced mass spectrometric methods applied to the study of fate and removal of pharmaceuticals in wastewater treatment, TrAC - Trends Anal. Chem. 26 (2007) 1132-1144.

[33] R. Grabic, J. Fick, R.H. Lindberg, G. Fedorova, M. Tysklind, Multi-residue method for trace level determination of pharmaceuticals in environmental samples using liquid chromatography coupled to triple quadrupole mass spectrometry, Talanta. 100 (2012) 183195.

[34] R. López-Serna, S. Pérez, A. Ginebreda, M. Petrović, D. Barceló, Fully automated determination of 74 pharmaceuticals in environmental and waste waters by online solid phase extraction-liquid chromatography- electrospray-tandem mass spectrometry, Talanta. 83 (2010) 410-424.

[35] S. Huntscha, H.P. Singer, C.S. McArdell, C.E. Frank, J. Hollender, Multiresidue analysis of 88 polar organic micropollutants in ground, surface and wastewater using online mixedbed multilayer solid-phase extraction coupled to high performance liquid chromatographytandem mass spectrometry, J. Chromatogr. A. 1268 (2012) 74-83. 
[36] T.A. Ternes, M.Bonerz, N. Herrmann, D. Löffler, E. Keller B.B. Lacida, A.C. Alder, Determination of pharmaceuticals, iodinated contrast media and musk fragrances in sludge by LC/tandem MS and GC-MS, J. Chromatogr. A. 1067 (2005) 213-223.

[37] A. Nieto, F. Borrull, E. Pocurull, R.M. Marcé, Pressurized liquid extraction: A useful technique to extract pharmaceuticals and personal-care products from sewage sludge, TrAC Trends Anal. Chem. 29 (2010) 752-764.

[38] A. Jelic, M. Gros, A. Ginebreda, R. Cespedes-Sánchez, F. Ventura, M. Petrovic, D. Barcelo, Occurrence, partition and removal of pharmaceuticals in sewage water and sludge during wastewater treatment, Water Res. 45 (2011) 1165-1176.

[39] B.F.D. Silva, A. Jelic, R. López-Serna, A.A. Mozeto, M. Petrovic, D. Barceló, Occurrence and distribution of pharmaceuticals in surface water, suspended solids and sediments of the Ebro river basin, Spain., Chemosphere. 85 (2011) 1331-1339.

[40] Y. Chen, Q. Cao, S. Deng, J. Huang, B. Wang, G. Yu, Determination of pharmaceuticals from various therapeutic classes in dewatered sludge by pressurized liquid extraction and high performance liquid chromatography and tandem mass spectrometry (HPLC-MS/MS), Int. J. Environ. Anal. Chem. DOI: 10.1080/03067319.2012.717271 (2012)

[41] O. Zuloaga, P. Navarro, E. Bizkarguenaga, A. Iparraguirre, A. Vallejo, M. Olivares, A. Prieto, Overview of extraction, clean-up and detection techniques for the determination of organic pollutants in sewage sludge: A review, Anal. Chim. Acta. 736 (2012) 7-29.

[42] J.-. Zhao, G.-. Ying, L. Wang, J.-. Yang, X.-. Yang, L.-. Yang, X. Li, Determination of phenolic endocrine disrupting chemicals and acidic pharmaceuticals in surface water of the Pearl Rivers in South China by gas chromatography-negative chemical ionization-mass spectrometry, Sci. Total Environ. 407 (2009) 962-974.

[43] V.G. Samaras, N.S. Thomaidis, A.S. Stasinakis, G. Gatidou, T.D. Lekkasa, Determination of selected non-steroidal anti-inflammatory drugs in wastewater by gas chromatography-mass spectrometry, Int. J. Environ. Anal. Chem. 90 (2010) 219-229.

[44] A. Sebok, A. Vasanits-Zsigrai, A. Helenkár, G. Záray, I. Molnár-Perl, Multiresidue analysis of pollutants as their trimethyl derivatives, by gas chromatography-mass spectrometry, J. Chromatogr. A. 1216 (2009) 2288-2301.

[45] N. Andrási, A. Helenkár, G. Záray, A. Vasanits, I. Molnár-Perl, Derivatization and fragmentation analysis of natural and synthetic steroids, as their trimethylsilyl (oxime) ether derivatives by gas chromatography mass spectrometry: Analysis of dissolved steroids in wastewater samples, J. Chromatogr. A. 1218 (2011) 1878-1890.

[46] D. Barceló, M. Petrovic, Challenges and achievements of LC-MS in environmental analysis: 25 years on, TrAC - Trends Anal. Chem. 26 (2007) 2-11.

[47] B. Petrie, E.J. McAdam, M.J. Whelan, J.N. Lester, E. Cartmell, The determination of nonylphenol and its precursors in a trickling filter wastewater treatment process, Anal. Bioanal. Chem. 405 (2013) 3243-3253. 
[48] S. Marchese, A. Gentili, D. Perret, G. D'Ascenzo, F. Pastori, Quadrupole time-of-flight versus triple-quadrupole mass spectrometry for the determination of non-steroidal antiinflammatory drugs in surface water by liquid chromatography/tandem mass spectrometry, Rapid Comm. Mass Spectr. 17 (2003) 879-886.

[49] A.C. Chiaia-Hernandez, M. Krauss, J. Hollender, Screening of lake sediments for emerging contaminants by liquid chromatography atmospheric pressure photoionization and electrospray ionization coupled to high resolution mass spectrometry, Environ. Sci. Technol. 47 (2013) 976-986.

[50] D.E. Helbling, J. Hollender, H.-.E. Kohler, H. Singer, K. Fenner, High-throughput identification of microbial transformation products of organic micropollutants, Environ. Sci. Technol. 44 (2010) 6621-6627.

[51] Y.-. Duan, C.-. Dai, Y.-. Zhang, Ling-Chen, Selective trace enrichment of acidic pharmaceuticals in real water and sediment samples based on solid-phase extraction using multi-templates molecularly imprinted polymers, Anal. Chim. Acta. 758 (2013) 93-100.

[52] K.H. Langford, M.D. Scrimshaw, J.W. Birkett, J.N. Lester, Degradation of nonylphenolic surfactants in activated sludge batch tests, Water Res. 39 (2005) 870-876.

[53] T. Urase, T. Kikuta, Separate estimation of adsorption and degradation of pharmaceutical substances and estrogens in the activated sludge process, Water Res. 39 (2005) 1289-1300.

[54] W.O. Khunjar, N.G. Love, Sorption of carbamazepine, 17 $\alpha$-ethinylestradiol, iopromide and trimethoprim to biomass involves interactions with exocellular polymeric substances, Chemosphere. 82 (2011) 917-922.

[55] C.D. Metcalfe, B.G. Koenig, D.T. Bennie, M. Servos, T.A. Ternes, R. Hirsch, Occurrence of neutral and acidic drugs in the effluents of canadian sewage treatment plants, Environ. Toxicol. Chem. 22 (2003) 2872-2880.

[56] A. Joss, E. Keller, A.C. Alder, A. Göbel, C.S. McArdell, T. Ternes, H. Siegrist, Removal of pharmaceuticals and fragrances in biological wastewater treatment, Water Res. 39 (2005) 3139-3152.

[57] M. Bernhard, J. Müller, T.P. Knepper, Biodegradation of persistent polar pollutants in wastewater: Comparison of an optimised lab-scale membrane bioreactor and activated sludge treatment, Water Res. 40 (2006) 3419-3428.

[58] N. Nakada, T. Tanishima, H. Shinohara, K. Kiri, H. Takada, Pharmaceutical chemicals and endocrine disrupters in municipal wastewater in Tokyo and their removal during activated sludge treatment, Water Res. 40 (2006) 3297-3303.

[59] O.A.H. Jones, N. Voulvoulis, J.N. Lester, The occurrence and removal of selected pharmaceutical compounds in a sewage treatment works utilising activated sludge treatment, Environ. Pollut. 145 (2007) 738-744. 
[60] K. Kimura, H. Hara, Y. Watanabe, Elimination of selected acidic pharmaceuticals from municipal wastewater by an activated sludge system and membrane bioreactors, Environ Sci. Technol. 41 (2007) 3708-3714.

[61] J. Radjenovic, M. Petrovic, D. Barceló, Analysis of pharmaceuticals in wastewater and removal using a membrane bioreactor, Anal. Bioanal. Chem. 387 (2007) 1365-1377.

[62] S. Zorita, L. Mårtensson, L. Mathiasson, Occurrence and removal of pharmaceuticals in a municipal sewage treatment system in the south of Sweden, Sci. Total Environ. 407 (2009) 2760-2770.

[63] M. Maurer, W. Pronk, T.A. Larsen, Treatment processes for source-separated urine Water Res. 40 (2006) 3151-3166.

[64] T.L. Zearley, R.S. Summers, Removal of trace organic micropollutants by drinking water biological filters, Environ. Sci. Technol. 46 (2012) 9412-9419.

[65] T.A. Ternes, J. Stüber, N. Herrmann, D. McDowell, A. Ried, M. Kampmann, B. Teiser, Ozonation: A tool for removal of pharmaceuticals, contrast media and musk fragrances from wastewater?, Water Res. 37 (2003) 1976-1982.

[66] S.D. Kim, J. Cho, I.S. Kim, B.J. Vanderford, S.A. Snyder, Occurrence and removal of pharmaceuticals and endocrine disruptors in South Korean surface, drinking, and waste waters, Water Res. 41 (2007) 1013-1021.

[67] V. Matamoros, C. Arias, H. Brix, J.M. Bayona, Removal of pharmaceuticals and personal care products (PPCPs) from urban wastewater in a pilot vertical flow constructed wetland and a sand filter, Environ. Sci. Technol. 41 (2007) 8171-8177.

[68] N. Nakada, H. Shinohara, A. Murata, K. Kiri, S. Managaki, N. Sato, H. Takada, Removal of selected pharmaceuticals and personal care products (PPCPs) and endocrine-disrupting chemicals (EDCs) during sand filtration and ozonation at a municipal sewage treatment plant, Water Res. 41 (2007) 4373-4382.

[69] S.A. Snyder, S. Adham, A.M. Redding, F.S. Cannon, J. DeCarolis, J. Oppenheimer, E.C. Wert, Y. Yoon, Role of membranes and activated carbon in the removal of endocrine disruptors and pharmaceuticals, Desalination. 202 (2007) 156-181.

[70] R. Rosal, A. Rodríguez, J.A. Perdigón-Melón, A. Petre, E. García-Calvo, M.J. Gómez, A. Agüera, A.R. Fernández-Alba, Occurrence of emerging pollutants in urban wastewater and their removal through biological treatment followed by ozonation, Water Res. 44 (2010) 578588.

[71] H. Schaar, M. Clara, O. Gans, N. Kreuzinger, Micropollutant removal during biological wastewater treatment and a subsequent ozonation step, Environ. Poll. 158 (2010) 1399-1404.

[72] D.P. Grover, J.L. Zhou, P.E. Frickers, J.W. Readman, Improved removal of estrogenic and pharmaceutical compounds in sewage effluent by full scale granular activated carbon: Impact on receiving river water, J. Hazard. Mater. 185 (2011) 1005-1011. 
[73] J. Reungoat, B.I. Escher, M. Macova, J. Keller, Biofiltration of wastewater treatment plant effluent: Effective removal of pharmaceuticals and personal care products and reduction of toxicity, Water Res. 45 (2011) 2751-2762.

[74] X. Yang, R.C. Flowers, H.S. Weinberg, P.C. Singer, Occurrence and removal of pharmaceuticals and personal care products (PPCPs) in an advanced wastewater reclamation plant, Water Res. 45 (2011) 5218-5228.

[75] M.M. Huber, S. Canonica, G.-. Park, U. Von Gunten, Oxidation of pharmaceuticals during ozonation and advanced oxidation processes, Environ Sci. Technol. 37 (2003) 10161024.

[76] L. Gunnarsson, M. Adolfsson-Erici, B. Björlenius, C. Rutgersson, L. Förlin, D.G.J. Larsson, Comparison of six different sewage treatment processes-Reduction of estrogenic substances and effects on gene expression in exposed male fish, Sci. Total Environ. 407 (2009) 5235-5242.

[77] A.O. Ifelebuegu, The fate and behavior of selected endocrine disrupting chemicals in full scale wastewater and sludge treatment unit processes Int. J. Environ. Sci. Technol. 8 (2011) 245-254.

[78] L. Ho, C. Grasset, D. Hoefel, M.B. Dixon, F.D.L. Leusch, G. Newcombe, C.P. Saint, J.D. Brookes, Assessing granular media filtration for the removal of chemical contaminants from wastewater, Water Res. 45 (2011) 3461-3472.

[79] A. Göbel, C.S. McArdell, A. Joss, H. Siegrist, W. Giger, Fate of sulfonamides, macrolides, and trimethoprim in different wastewater treatment technologies, Sci. Total Environ. 372 (2007) 361-371.

[80] T. Hashimoto, K. Takahashi, T. Murakami, Characteristics of estrogen decomposition by ozonation, Water Sci. Technol. 54 (2006) 87-93.

[81] M. Wagner, N.P. Ivleva, C. Haisch, R. Niessner, H. Horn, Combined use of confocal laser scanning microscopy (CLSM) and Raman microscopy (RM): Investigations on EPS Matrix, Water Res. 43 (2009) 63-76.

[82] O.A.H. Jones, N. Voulvoulis, J.N. Lester, Human pharmaceuticals in wastewater treatment processes, Crit. Rev. Environ. Sci. Technol. 35 (2005) 401-427.

[83] D.B. Wunder, V.A. Bosscher, R.C. Cok, R.M. Hozalski, Sorption of antibiotics to biofilm, Water Res. 45 (2011) 2270-2280.

[84] M. Hörsing, A. Ledin, R. Grabic, J. Fick, M. Tysklind, J.L.C. Jansen, H.R. Andersen, Determination of sorption of seventy-five pharmaceuticals in sewage sludge, Water Res. 45 (2011) 4470-4482.

[85] J. Gong, Y. Ran, D. Chen, Y. Yang, E.Y. Zeng, Association of endocrine-disrupting chemicals with total organic carbon in riverine water and suspended particulate matter from the Pearl River, China., Environ. Toxicol. Chem. 31 (2012) 2456-2464. 
[86] G. Guibaud, S. Comte, F. Bordas, S. Dupuy, M. Baudu, Comparison of the complexation potential of extracellular polymeric substances (EPS), extracted from activated sludges and produced by pure bacteria strains, for cadmium, lead and nickel, Chemosphere. 59 (2005) 629-638.

[87] J.P. Bagnall, A. Ito, E.J. McAdam, A. Soares, J.N. Lester, E. Cartmell, Resource dependent biodegradation of estrogens and the role of ammonia oxidising and heterotrophic bacteria, J. Hazard Mater. 239-240 (2012) 56-63.

[88] L.S. Gaulke, S.E. Strand, T.F. Kalhorn, H.D. Stensel, 17 $\alpha$-ethinylestradiol transformation via abiotic nitration in the presence of ammonia oxidizing bacteria, Environ. Sci. Technol. 42 (2008) 7622-7627.

[89] J.S. Vader, C.G. Van Ginkel, F.M.G.M. Sperling, J. De Jong, W. De Boer, J.S. De Graaf, M. Van Der Most, P.G.W. Stokman, Degradation of ethinyl estradiol by nitrifying activated sludge, Chemosphere. 41 (2000) 1239-1243.

[90] T. Kosjek, E. Heath, S. Pérez, M. Petrović, D. Barceló, Metabolism studies of diclofenac and clofibric acid in activated sludge bioreactors using liquid chromatography with quadrupole - time-of-flight mass spectrometry, J. Hydrology. 372 (2009) 109-117.

[91] S. Carbonaro, M.N. Sugihara, T.J. Strathmann, Continuous-flow photocatalytic treatment of pharmaceutical micropollutants: Activity, inhibition, and deactivation of $\mathrm{TiO} 2$ photocatalysts in wastewater effluent, Applied Catalysis B: Environmental. 129 (2013) 1-12.

[92] J.L. Chen, S. Ravindran, S. Swift, L.J. Wright, N. Singhal, Catalytic oxidative degradation of 17 $\alpha$-ethinylestradiol by FeIII-TAML/H202: Estrogenicities of the products of partial, and extensive oxidation, Water Res. 46 (2012) 6309-6318.

[93] M.M. Huber, T.A. Ternes, U. Von Gunten, Removal of estrogenic activity and formation of oxidation products during ozonation of $17 \alpha$-ethinylestradiol, Environ. Sci. Technol. 38 (2004) 5177-5186.

[94] J. Hoigne, H. Bader, Rate constants of reactions of ozone with organic and inorganic compounds in water. I. Non-dissociating organic compounds, Water Res. 17 (1983) 173-183.

[95] S.A. Snyder, P. Westerhoff, Y. Yoon, D.L. Sedlak, Pharmaceuticals, personal care products, and endocrine disruptors in water: Implications for the water industry, Environ. Eng. Sci. 20 (2003) 449-469.

[96] A.D. Coelho, C. Sans, A. Agüera, M.J. Gómez, S. Esplugas, M. Dezotti, Effects of ozone pre-treatment on diclofenac: Intermediates, biodegradability and toxicity assessment, Sci. Total Environ. 407 (2009) 3572-3578.

[97] C.-. Chiu, K. Hristovski, S. Huling, P. Westerhoff, In-situ regeneration of saturated granular activated carbon by an iron oxide nanocatalyst, Water Res. 47 (2013) 1596-1603.

[98] Environment Agency, Endocrine Disrupting Substances in the Environment: The Environment Agency's Strategy, Environment Agency, HMSO, London, UK. 2000. 
[99] D.R. Baker, B. Kasprzyk-Hordern, Critical evaluation of methodology commonly used in sample collection, storage and preparation for the analysis of pharmaceuticals and illicit drugs in surface water and wastewater by solid phase extraction and liquid chromatography-mass spectrometry, J. Chromatogr. A. 1218 (2011) 8036-8059.

[100] J. Shen, X. Jin Yang, A.I. Schäfer, Quantification of hormone-humic acid interactions in nanofiltration, Environ. Sci. Technol. 46 (2012) 10597-10604. 